Thierry Aubry, Luís Luís

\title{
Umwelt und sozialer Kontext der paläolithischen Freilandkunst im Côa-Tal (Portugal)*
}

Upper Palaeolithic authorship of open-air rock art is a fairly recent discovery. After Altamira and cave art, the discovery of Côa Valley rock art and its archaeological context was pivotal in the process by which this new reality achieved scientific recognition. Although stylistically similar and representing the same general species (mostly horses, aurochs, ibex and deer), these images were inscribed in rock panels distributed within the same territories exploited by their forager authors. Interpretation of why certain surfaces were chosen and what function these images served must be based on the study of the geological nature of the engraved panels, dating of the engravings and their subsequent evolution. Once these factors have been evaluated, it will be possible to interpret the distribution of the Côa Valley's two most important phases (pre-Magdalenian and Lateglacial) and consider their visibility and possible spatial relations. Our final goal will be to try to understand this rock art in its social contexts.

Im Jahre 1981 wurde eine in Mazouco, im Nordosten Portugals, entdeckte Gravierung, die ein Pferd darstellt (Abb. 1), mit den Bildern verglichen, die man seit mehr als einem Jahrhundert auf Wänden von Höhlen und Abris sowie auf beweglichen Gegenständen zu entdecken gewohnt war1. Implizit wurde damit erstmals angenommen, dass Gravierungen paläolithischen Alters sich auch auf unmittelbar einer Beeinträchtigung durch die Verwitterung ausgesetzten Felsen erhalten können. Diese Entdeckung und ihre zeitliche Einordnung ebneten den Weg für weitere Funde (Abb. 1) in Spanien ${ }^{2}$ und an der östlichen Grenze des französischen Teils der Pyrenäen ${ }^{3}$.

15 Jahre später führte der Konflikt um den von der Elektrizitätsgesellschaft EDP (Energias de Portugal) geplanten Bau eines Staudammes und die deshalb drohende Überflutung von Felsbildstationen des unteren Côa-Tales ${ }^{4}$ zur Entdeckung weiterer Fundplätze in Portugal - im Tal des Sabor, des Ocreza ${ }^{5}$ und des Zêzere ${ }^{6}$-, in Spanien - in Molino Manzanez ${ }^{7}$ - sowie, fast ein Jahrhundert nach bekanntwerden der Höhlenkunst, zur Anerkennung der Existenz einer paläolithischen Freilandkunst, und zwar sowohl durch die Prähistoriker als auch durch einen geringen Teil des breiten Publikums.

Diese Bilder befinden sich auf ebenen, senkrechten oder seltener nahezu waagerechten Oberflächen im Bereich der Aufschlüsse metamorpher Gesteine alter Gebirgsstöcke,

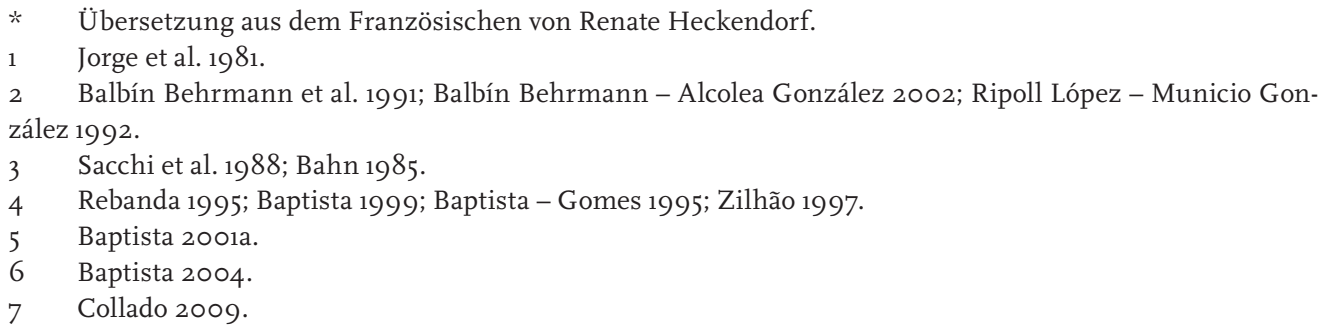




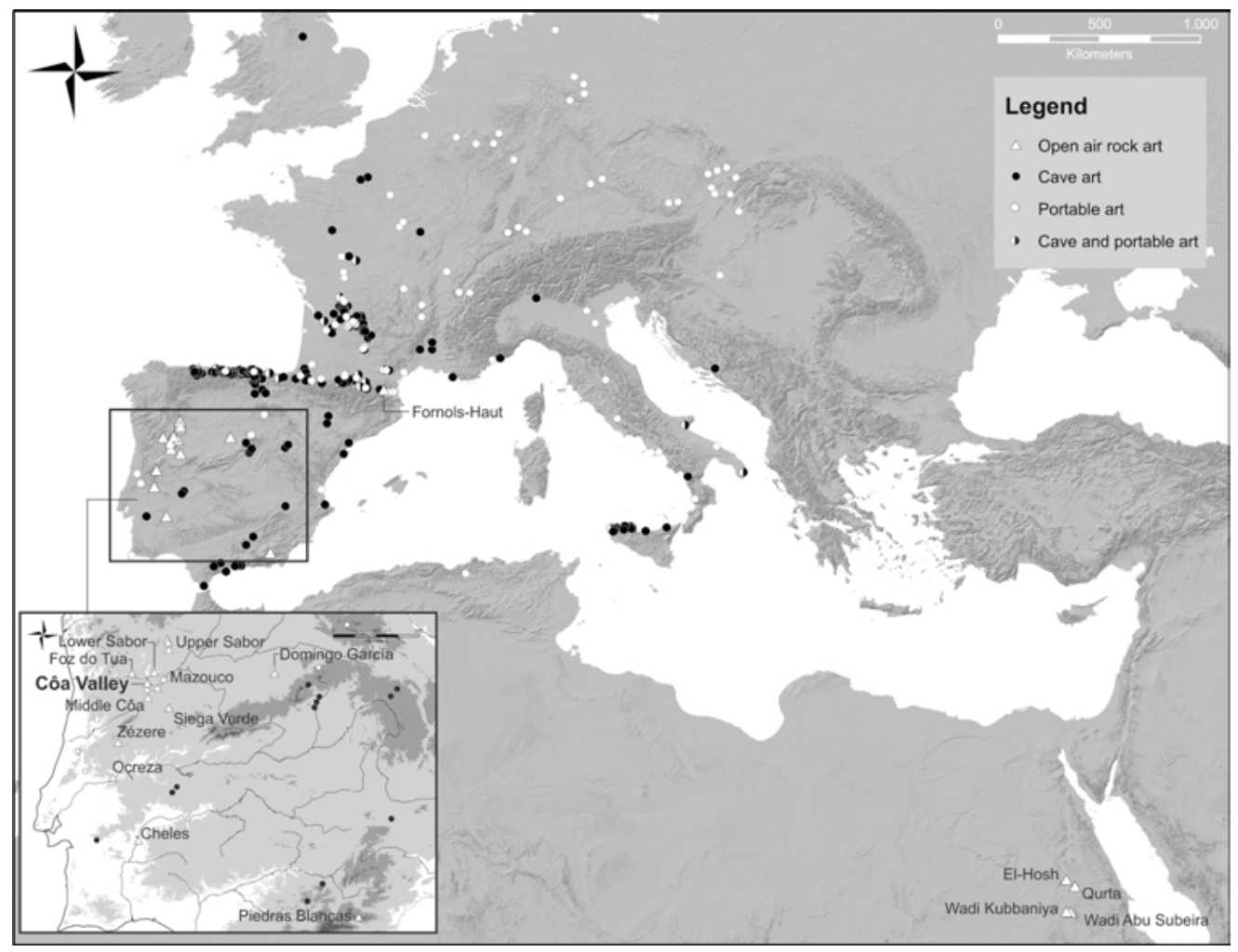

Abb. 1 | Lagebestimmungskarte der unterschiedlichen Typen jungpaläolithischer Zeichnungen.

die das Gerüst der Iberischen Halbinsel und der Pyrenäen-Kette bilden (Abb. 1). Sie sind in klimatischen Umgebungen erhalten, in denen die Verwitterung der Aufschlüsse unter dem Einfluss des Klimawandels des letzten glazialen Maximums und des Spätglazials auf den ersten Blick weniger stark als die periglazialen Prozesse nördlich der Pyrenäen ${ }^{8}$ ausgeprägt war.

Die Freilandkunst ist gedanklichen Prozessen geschuldet, die sich - durch die symbolische Aneignung des Territoriums durch das Bild und dessen Fortbestand - von dem in den Tiefen der Höhlen hinterlassenen graphischen Ausdruck unterscheiden`. Während die Höhlenarchitektur von grundlegender Bedeutung für die Interpretation der darin erhaltenen Bilder ist, erlangen im Fall der Freilandkunst Merkmale wie die Sichtbarkeit und die Beziehung zur Umwelt eine andere Bedeutung und werfen neue Fragen auf. 15 Jahre nach der Entdeckung der Gravierungen des Côa-Tales möchten wir eine von 
Abb. 2a | Lagebestimmung der Freilandfundstellen von Felsbildern und Besiedlungsspuren im Côa-Tal.

Abb. 2b| Topographische Position der Freilandfundstellen im Verhältnis zum Wasserlauf.
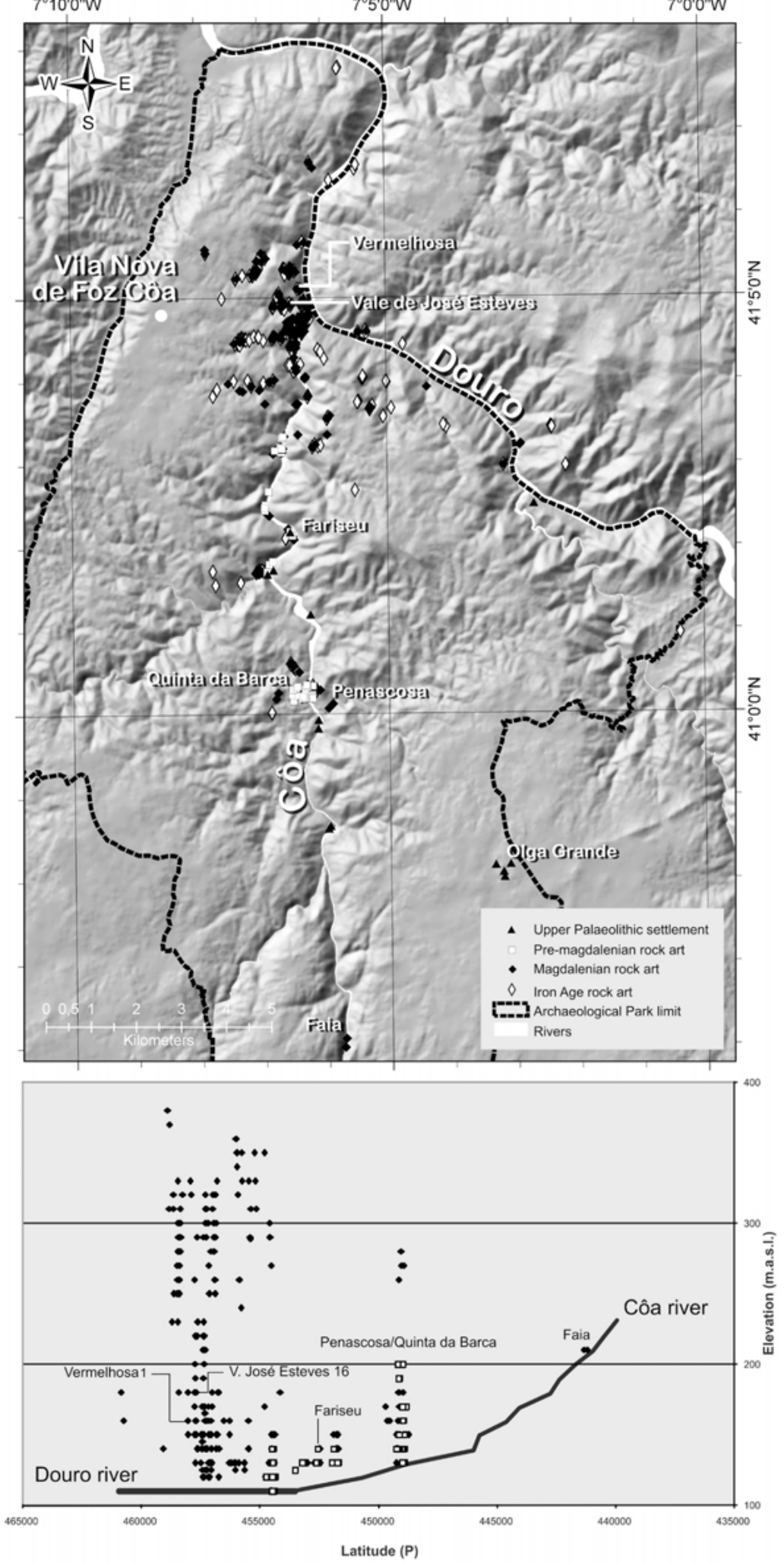
uns entwickelte Methodik vorstellen, mit der eine Deutung der Bilder und der sonstigen Überreste, die innerhalb der von ihren Urhebern in mehreren Phasen des Paläolithikums aufgesuchten Territorien erhalten sind, möglich ist.

\section{Die Kunst des Côa-Tales}

Bei den als »Kunst des Côa-Tales« bezeichneten Hinterlassenschaften handelt es sich um ca. 30 Fels-Konzentrationen, die Petroglyphen oder seltener mit mineralischen Pigmenten ausgeführte Zeichnungen tragen und sich auf die letzten 17 Kilometer des Côa-Flusses und der unmittelbar oberhalb und unterhalb seiner Einmündung in den Douro gelegenen Nebenflüsse verteilen (Abb. 2) ${ }^{10}$. Im Jahre 1998 wurde dieses Ensemble, dessen erste Bestandteile im Jahre 1991 - im Zusammenhang mit dem heute aufgegebenen Staudammprojekt - entdeckt wurden, in die Weltkulturerbeliste der Unesco aufgenommen. Als Ergebnis der seither durchgeführten Begehungen konnte eine Liste mit etwa 1000 Bildfeldern erstellt werden, die Petroglyphen aus allen Perioden tragen, wobei 330 dieser Felsoberflächen Motive aufweisen, die nach stilistischen Kriterien dem Paläolithikum zuzuordnen sind ${ }^{11}$.

Die Bilder wurden im Verlauf eines über 20.000 Jahre andauernden Zyklus, der im Jungpaläolithikum begann und sich dann bis in das 20. Jahrhundert hinein fortsetzte, an den Felswänden angebracht. Die ältesten Darstellungen zeigen bestimmte, von ihren Urhebern gejagte Tiere (Pferde, Auerochsen, Steinböcke, Hirsche, Pyrenäen-Gämsen etc.; Abb. 4). Gegen Ende des Paläolithikums kommen menschliche Darstellungen und Zeichen zu den Tierbildern hinzu ${ }^{12}$. Die eisenzeitlichen Petroglyphen zeigen mythologische Szenen sowie Kriegs- und Jagdszenen (Krieger, Waffen, Pferde, Rothirsche, Hunde) ${ }^{13}$. Die jüngsten, häufig datierten Zeichnungen bilden religiöse Gegenstände oder alltägliche, von ihren Urhebern - den Müllern, die das Tal in den 1950er Jahren aufgegeben haben beobachtete Begebenheiten $\mathrm{ab}^{14}$.

Im Lauf der Zeit ist ein Wandel der Techniken, die die > Verewigung < der Bilder ermöglicht haben, zu beobachten (Abb. 3). Grundsätzlich ging es darum, auf einer ebenen und, in Abhängigkeit von der Expositionsdauer, braunen oder dunkelgrauen Oberflächen aus Schiefer- oder Grauwacke einen Kontrast zu erzeugen. Der Strich besteht zumeist aus feinen während des Paläolithikums mit Steinwerkzeugen und von der Eisenzeit an mit Metallgerätschaften erzeugten Ritzlinien. In anderen Fällen entstanden durch wiederholte Punzund Ritzvorgänge, gelegentlich auch durch Abschaben [Anm. d. Übers.: bzw. Abschleifen] der Felsoberfläche, tief eingeschnittene Linien mit U- oder V-förmigem Querschnitt.

10 Zilhão 1997; Baptista 2001b; Baptista - García Diez 2002; Baptista et al. 2006; Baptista - Reis 2008.

11 Baptista - Reis 2008.

12 Baptista 1999; Baptista 2009.

13 Luís 2009.

14 García Diez - Luís 2003. 

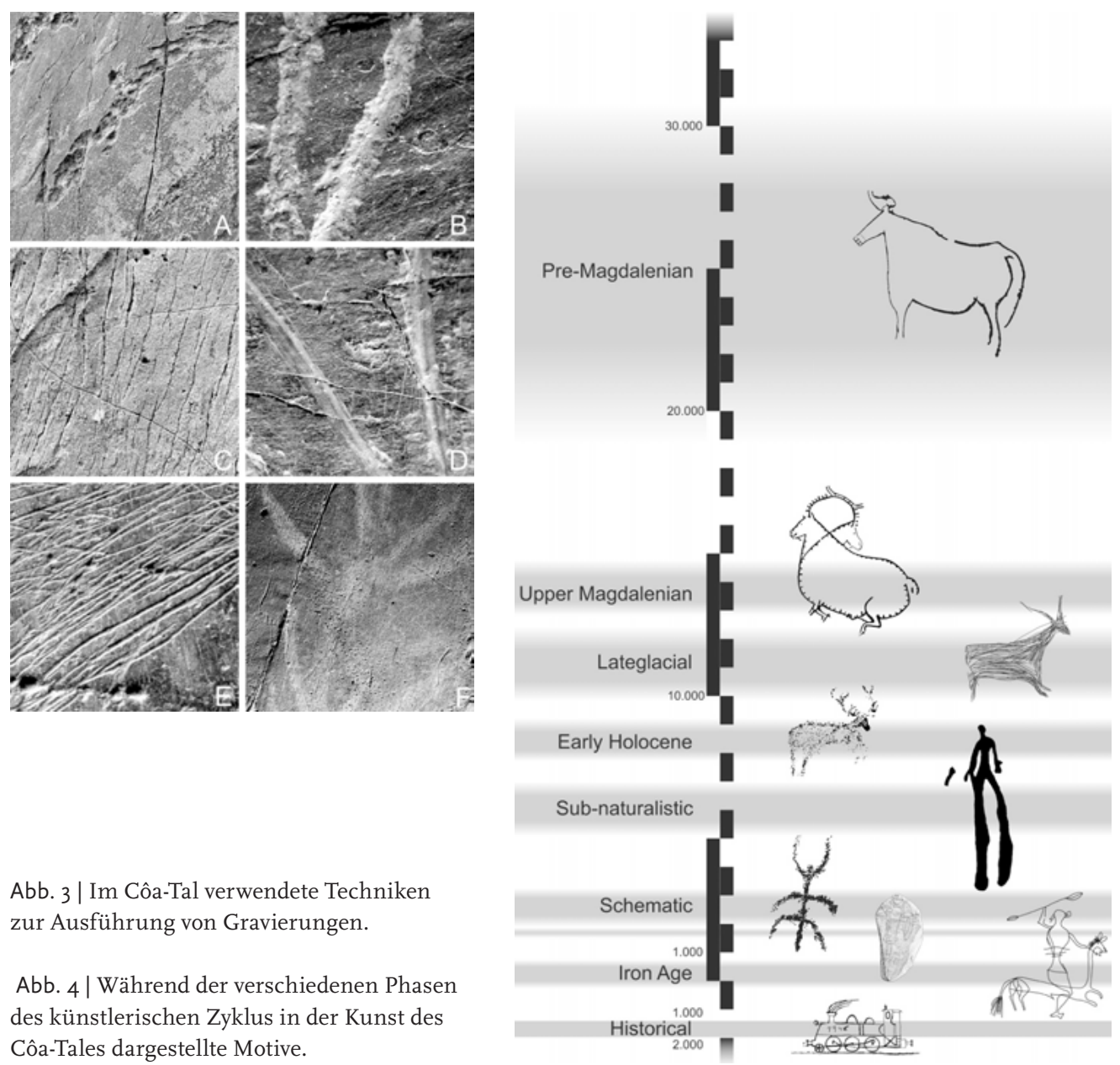

Abb. 3 | Im Côa-Tal verwendete Techniken zur Ausführung von Gravierungen.

Abb. 4 | Während der verschiedenen Phasen des künstlerischen Zyklus in der Kunst des Côa-Tales dargestellte Motive.

Die an den paläolithischen Fundorten der Region durchgeführten Ausgrabungen erbrachten in einer um 28.500 BP datierten Schicht der Fundstelle Olga Grande $4^{15}$ die Entdeckung von Grobspitzen, deren Enden Schäden aufweisen, die einer Verwendung zur Erzeugung von Punzungen und Rillen entsprechen ${ }^{16}$. Obwohl die Ausgrabungen der jungpaläolithischen Fundplätze der Region zahlreiche Fragmente mineralischer Pigmente aus unterschiedlichen regionalen Quellen zu Tage gefördert haben ${ }^{17}$, war hinsichtlich der gravierten Felsbildlinien nur in denen der Fundstelle Faia stellenweise die Erhaltung mineralischer Pigmente nachweisbar.

$15 \quad$ Valladas et al. 2001; Aubry 2009.

16 Plisson 2009.

17 García Diez et al. 2009. 


\subsection{Aus welcher Zeit stammen die im Côa-Tal erhaltenen paläolithischen Petroglyphen?}

Bereits zum Zeitpunkt der Entdeckung der Côa-Gravierungen waren sich die Prähistoriker darüber einig, dass ein Teil der Felsbilder in keiner Weise den Zeichen oder den schematisierten anthropomorphen und zoomorphen Motiven der Bauern und Hirten des Neolithikums oder der jüngeren Perioden ähnelt, sondern den durch paläolithische Jäger in Höhlen und Abris hinterlassenen, gemalten und in Stein geschlagenen Tierdarstellungen entspricht.

Die EDP (Energias de Portugal) versuchte unter Berufung auf einige abweichende Stimmen, die Geltung des stilistischen Vergleiches [Anm. d. Übers.: grundsätzlich] in Frage zu stellen. Anhand von Kleinstbruchstücken des organischen Materials, das in den Felsbildlinien einiger Darstellungen an den Fundorten Penascosa, Piscos und Canada do Inferno erhalten war, wurde mit Hilfe der Radiokarbonmethode eine Altersbestimmung vorgenommen. Die Ergebnisse liegen nach Alan Watchman ${ }^{18}$ zwischen 2000 und 7000 , nach Ron Dorn ${ }^{19}$ zwischen 2120 und 5480 Jahre vor heute. Gleichwohl reichen die durch Dorn vorgenommenen Datierungen von Kleinstbruchstücken organischen Materials, das sich auf den an die Gravierungen angrenzenden Oberflächen erhalten hat, im Fall einer Probe vom Fundort Penascosa etwa 29.990 Jahre zurück. Unter den beiden zitierten Autoren ist die Bedeutung der Ergebnisse der Datierungen und deren Verwendbarkeit zur Bestimmung der zeitlichen Abfolge der Herstellung bzw. Auffrischung der Gravierungen strittig. Auf der Grundlage einer vergleichenden Betrachtung der an Kleinstbruchstücken organischen Materials (sowohl aus den Felsbildlinien als auch von angrenzenden Oberflächen) vorgenommenen Datierungen schließt Dorn ${ }^{20}$ - ebenso wie João Zilhão ${ }^{21}$ es bereits auf der Basis theoretischer Überlegungen erklärt hatte -, dass die Verwitterungsrinde der Felsen nicht als geschlossenes System anzusehen ist und folglich, aufgrund der Besiedlung durch Mikroorganismen, seit dem Zeitpunkt der Erstexposition die Felsoberfläche einer fortwährenden Verunreinigung ausgesetzt gewesen war. Deshalb ist nicht davon auszugehen, dass die Datierungsergebnisse den Herstellungszeitpunkt der Felsbildlinien angeben.

In Anbetracht der Tatsache, dass sich die Gravierungen mittels der Radiokarbonmethode nicht datieren lassen, wurde schnell klar, dass eine Bestimmung ihres archäologischen Kontextes notwendig war. Aus den an rund 15 jungpaläolithischen Stationen - von denen zehn sondiert oder ausgegraben werden konnten - erhaltenen Abfolgen ist seither der genaue Rahmen der Besiedlung der Region ersichtlich ${ }^{22}$. Die seit Beginn der Forschungen auf der Grundlage typologischer und technologischer Erwägungen vorgebrach-

Dorn 1997.

Zilhão 1995 .

Aubry 2001; Aubry - Sampaio 2008; Aubry 2009. 


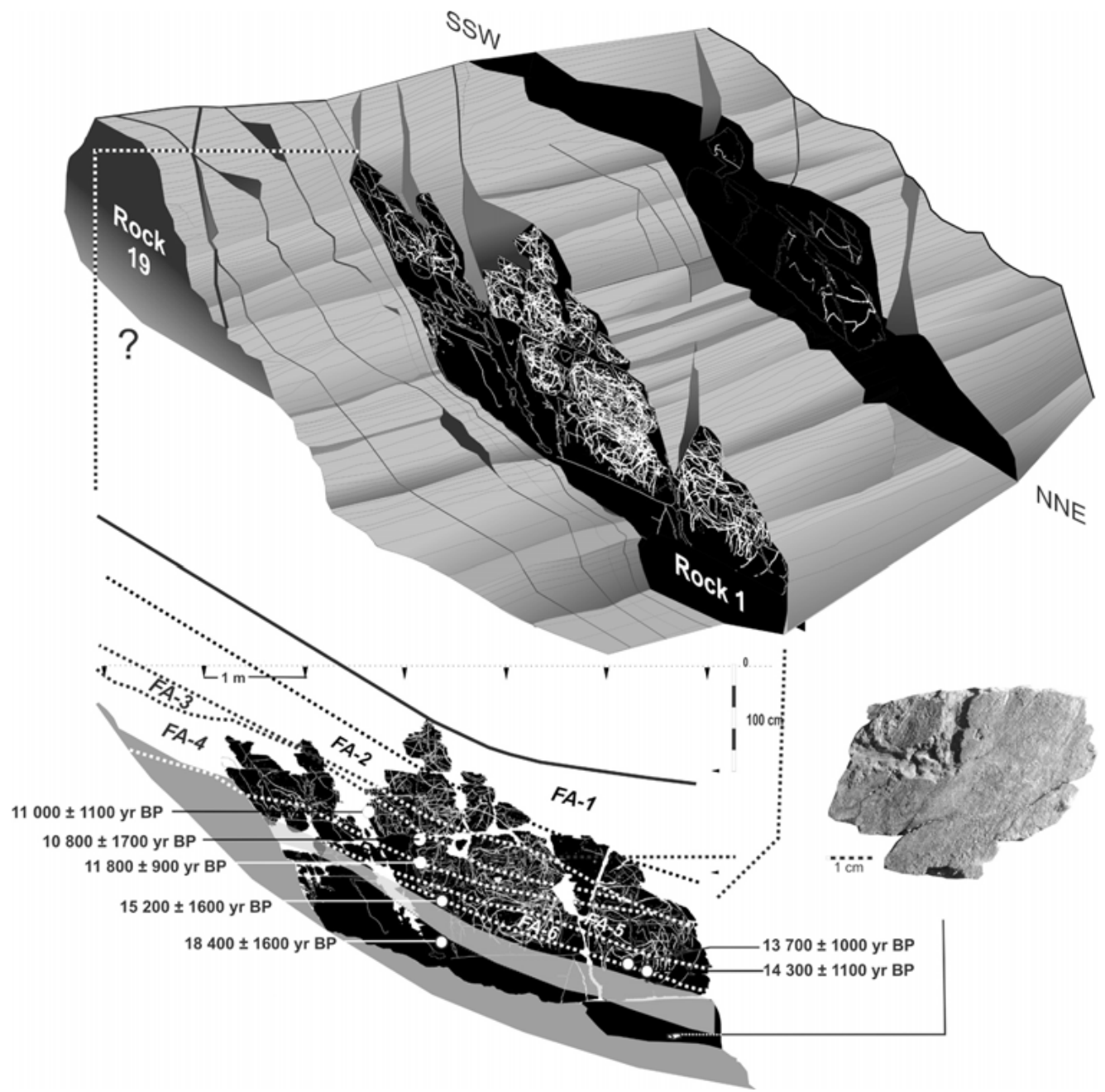

Abb. 5 Rekonstruktion der den Felsen Nr. 1 des Fundortes Fariseu umgebenden Felsaufschlüsse und Überlagerung der Darstellungen durch datierte Schichten, die jungpaläolithische Besiedlungsreste enthielten.

ten Thesen über die menschliche Besiedlung des Côa-Tales während mehrerer Phasen des Jungpaläolithikums ${ }^{23}$ konnten durch Thermolumineszenzdaten bestätigt werden ${ }^{24}$, so dass sich auch die von Bednarik ${ }^{25}$ vorgebrachte Argumentation hinsichtlich eines fehlenden Kontextes widerlegen ließ.

Im Jahre 1999 konnte am Fundort Fariseu das paläolithische Alter der Gravierungen nachgewiesen werden. Dort bedeckten Schichten mit jungpaläolithischen Besiedlungs-

23 Zilhão 1995; Zilhão 1997; Aubry 1998; Aubry 2001.

24 Mercier et al. 2001; Valladas et al. 2001; Mercier et al. 2006.

25 Bednarik 1995. 
resten eine Wand, die mit stilistisch dieser Periode zugehörigen Motiven verziert war. Der Fundplatz lieferte eine stratigraphische Abfolge mit Überresten mehrerer jungpaläolithischer Besiedlungsphasen. Die Stratigraphie befand sich im direkten Kontakt mit einer Felswand, die 94 Tierdarstellungen trägt (Abb. 5). Diese durch Punzung, tiefe Schlifflinien [Anm. d. Übers.: im Originaltext ist von »Rillen« die Rede] oder seltener Ritzung erzeugten Bilder zeigen Pferde, Hirsche, Rinder und Ziegenartige (Abb. 4. 5) ${ }^{26}$. Am Ausgangspunkt der ausgegrabenen Sequenz fand sich ein von der bearbeiteten Felswand herabgefallenes Bruchstück. Der archäologische Befund - d. h. zum einen die die bearbeitete Felswand bedeckende stratigraphische Abfolge und zum anderen die Überlagerungssequenz der Felsbilder - zeigt, dass von Anfang an - d. h. nach der verfügbaren OSL-Datierung seit mindestens $18.400 \pm 1600$ BP, was einer Radiokarbondatierung von 14.500/15.000 Jahren entspricht ${ }^{27}$ - die gesamte verfügbare Felsoberfläche genutzt wurde.

Diesem Befund nach zu urteilen gehen die Bilder des Côa-Tales, die mehrere übereinstimmende formale Merkmale aufweisen - Profildarstellung von Tieren durch die Verknüpfung gerader, geometrischer, durch tiefe Punzung und/oder Schlifflinien [Anm. d. Übers.: im Originaltext ist von »Rillen« die Rede $]^{28}$ erzeugter Linien, wobei keine Einzelheiten des Körpers und die vorderen und hinteren Gliedmaßen jeweils nur einfach und ohne das Extremitätenende abgebildet sind - zeitlich dem Datum von 18.400 BP voraus und sind älter als die Magdalénien-Kultur. Eine im Jahre 2007 im Mittelpunkt des Fundortes durchgeführte Sondierungsgrabung führte zur Entdeckung älterer Besiedlungsspuren, die - aufgrund der Datierungsergebnisse von Holzkohleproben, die ein Alter von $19.020 \pm 80$ BP (GrA 40167) ergaben - dem Solutréen und - aufgrund der Merkmale der lithischen Industrie - dem Gravettien zuzuordnen sind. Dieser Befund deutet darauf hin, dass die Ausarbeitung der gepunzten und geschabten [Anm. d. Übers.: im Originaltext ist von »Rillen« die Rede] Felsbildsequenz des Felsens Nr. 1 älter als oder zeitgleich mit dem >Oberen Solutréen< ist, dass im Übrigen an mehreren Fundstellen der Region nachgewiesen werden konnte $^{29}$.

\subsection{Chronologische Abfolge auf der Grundlage von Überresten der Kleinkunst}

Während die Ausgrabungen am Fundort Fariseu einen Mindestzeitansatz für die frühe Kunstphase des Paläolithikums im Côa-Tal ergaben, führten die archäologischen Ausgrabungen an anderen Fundplätzen zur Entdeckung graphischer Zeugnisse auf beweglichem steinernen Trägermaterial, anhand derer sich die stilchronologische Abfolge näher bestimmen lässt (Abb. 6). Die Fundstelle Quinta da Barca Sul erbrachte einen schiefernen

26 Aubry - Baptista 2000; Aubry - García Diez 2000; Baptista 2009.

27 Aubry - Sampaio 2008; Aubry 2009.

28 Guy 2010.

29 Zilhão 2003; Aubry 2009. 
Abb. 6 | Chronologischer Rahmen der Zeichnungen auf beweglichen steinernen Bildträgern aus den paläolithischen Fundorten des Côa-Tales.
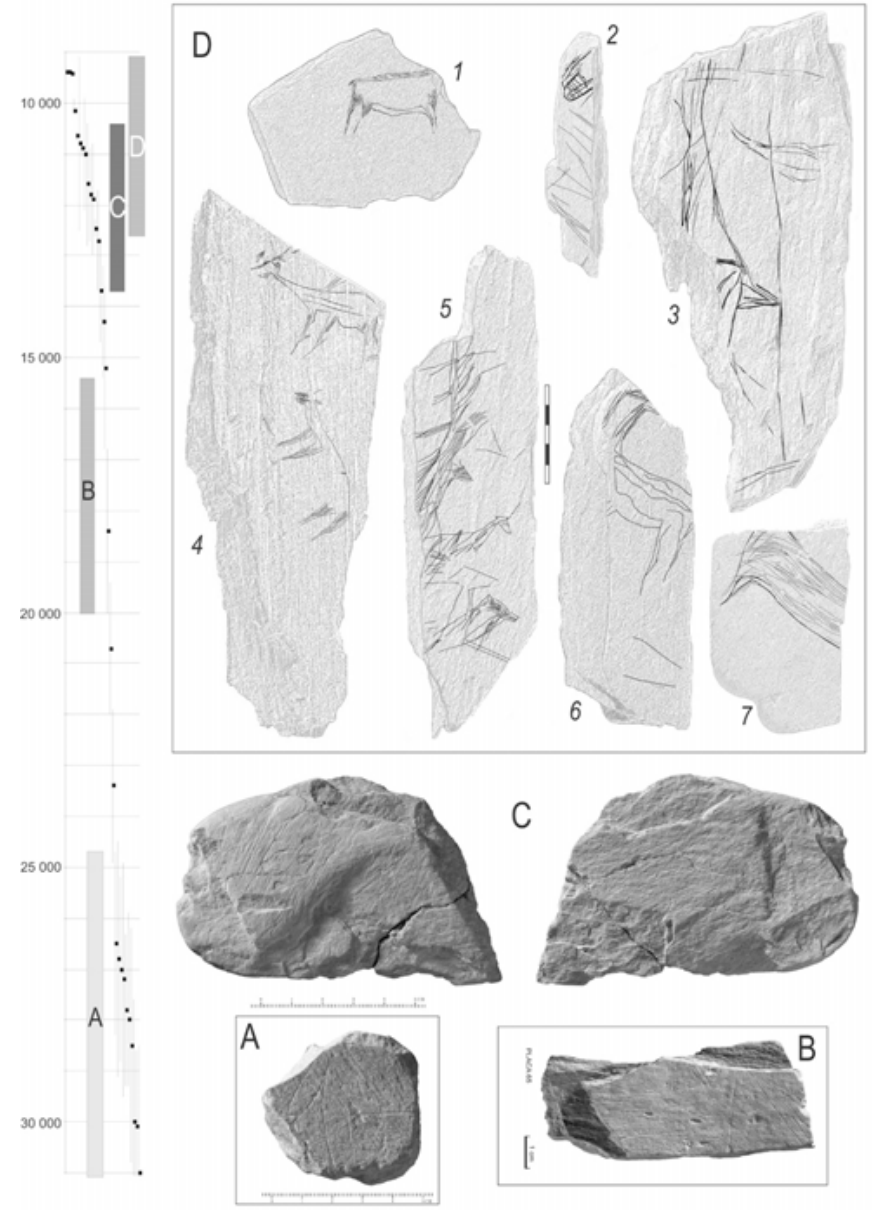

Kieselstein, der auf beiden Seiten Bündel paralleler Ritzlinien trägt und somit definitionsgemäß den >Azilien-Kieseln $<$ zuzurechnen ist ${ }^{30}$. Diese stilistische Einordnung wird durch das in der stratigraphischen Einheit Nr. 3, aus der dieses Objekt stammt, gefundene lithische Material und die TL-Datierung dreier erhitzter Quartz-Kiesel (11.600 $\pm 1.200,11.900$ \pm 1.100 und $12.700 \pm 100 \mathrm{BP}$ ) aus diesem Befund gestützt.

$\mathrm{Zu}$ den ersten beiden Exemplaren, die im Rahmen der im Jahre 1999 am Fels Nr. 1 des Fundortes Fariseu durchgeführten Sondierungsgrabung in der Schicht Nr. 4 gefunden wurden ${ }^{31}$, sind mehr als 80 Stücke hinzugekommen. Unter diesen im Jahre 2004 bei geologischen und geophysikalischen Sondierungen sowie in den Jahren 2005 und 2007

31 García Diez - Aubry 2002. 
bei Ausgrabungen entdeckten Objekten weisen rund 50 zweifellos anthropogene Ritzungen auf ${ }^{2}$. Zwei Exemplare mit anthropogenen Zeichnungen stammen aus einem Bereich, in dem sich die stratigraphischen Einheiten Nr. 7 und Nr. 8 nicht voneinander unterscheiden lassen. Obgleich sie unvollständig sind, zeigt jedes dieser Stücke ein unter stilistischen Gesichtspunkten charakteristisches Motiv. Alle übrigen gravierten Schieferstücke stammen aus der stratigraphischen Einheit Nr. 4. Aufgrund der TL-Datierungen (11.000 $\pm 1.000,10.800 \pm 1.700$ und $11.800 \pm 900)$ sowie der Datierung der Knochen $(10.510 \pm 40$ et $9.830 \pm 130 \mathrm{BP}$ ), die dem organischen Material der Schicht Nr. 4 zugehören, ist die Ausarbeitung dieser zoomorphen Motive in die Kaltphase der älteren Dryaszeit einzuordnen und somit zeitgleich mit der Besiedlungsphase an der Basis der Schicht Nr. 3 vom Fundplatz Quinta da Barca Sul. Bezüglich morphologischer und stilistischer Aspekte unterscheiden sich die an den zoomorphen Figuren aus der Schicht Nr. 4 erkennbaren Darstellungskonventionen nicht von denen, die schon bei den ersten Fundobjekten beobachtet wurden ${ }^{33}$. Die von Fernando Barbosa und André Santos vorgelegten Beschreibungen und systematischen Aufnahmen zeigen, dass zur Erzeugung der einzelnen Linien, die man in Parallelsequenzen anordnete oder zu figurativen bzw. zoomorphen Darstellungen zusammenfügte, feine Ritzungen als Technik angewandt wurden. Unter den zoomorphen Abbildungen dominieren Hirsche, Ziegenartige oder Gämsen. In einem Fall handelt es sich möglicherweise um eine anthropomorphe Figur oder um die Darstellung eines nachtaktiven Raubvogels in Vorderansicht. Die Wiedergabe des Körpers der zoomorphen Figuren gründet sich auf dieselben geometrischen Konstruktionsprinzipien, die bereits an den im Jahre 1999 entdeckten Objekten aufgefallen waren, wobei an den jeweils zwei oder vier abgebildeten Gliedmaßen das Extremitätenende nicht dargestellt ist (Abb. 6).

Der Vergleich der Kleinkunstfunde aus der Schicht 4 vom Fundort Fariseu mit den Zeichnungen an den Felswänden des unteren Côa-Tales und der Einmündung in den Douro zeigt große stilistische Übereinstimmungen ${ }^{34}$. Auf dieser Grundlage lassen sich zahlreiche durch Mehrfachritzungen erzeugte Bilder von Hirschen und Ziegenartigen in diese Endphase des Pleistozäns einordnen (Abb. 7). Weil genau diese Technik auch auf einem Fundstück aus der Schicht Nr. 7/8 verwendet wurde, kann allerdings nicht ausgeschlossen werden, dass diese stilistische Tradition in einer dem Magdalénien vorausgehenden Phase begonnen hat.

Im stratigraphischen Zusammenhang mit den Objekten, die gegenständliche Motive aufweisen, wurde auch ein Quarzit-Kiesel gefunden, der auf beiden Seiten die Reste mineralischer, in parallelen Streifen angeordneter Pigmente träg ${ }^{35}$. Dieses Motiv ist Bestandteil der Bildervielfalt der >Azilien-Kiesel ${ }^{36}$.

32 Aubry 2009.

33 García Diez - Aubry 2002.

34 Baptista 2008.

35 Aubry 2009.

36 D'Errico 1994; Couraud 1985. 

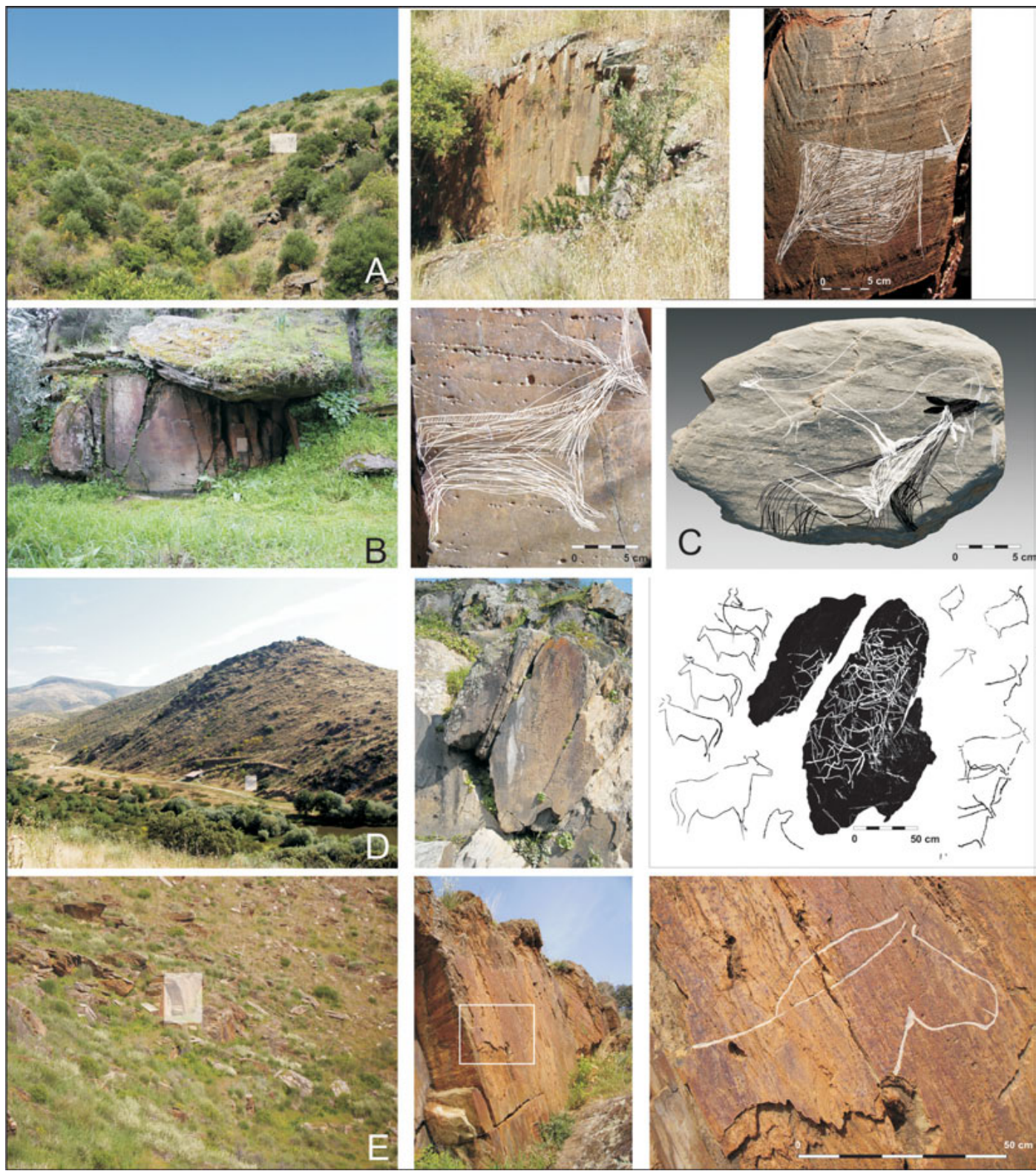

Abb. 7| Stilkonventionen der älteren und jüngeren Phasen des Jungpaläolithikums. 


\section{Interpretation der paläolithischen Petroglyphen}

\subsection{Was stellen die paläolithischen Petroglyphen dar?}

Die Tierdarstellungen überwiegen bei weitem - wie es auch im restlichen in den Höhlen und Abris des Côa-Tales erhaltenen Bilderkorpus und während der verschiedenen Kunstphasen der Fall ist - und im Bestiarium dominieren die Pferde, die großen Rinder (im vorliegenden Fall der Auerochse) und in geringerem Maße die Gämsen. Gleichwohl zeigt der Vergleich zwischen den beiden anerkannten Hauptphasen (d. h. einerseits die durch die Bilder auf dem Felsen Nr. 1 von Fariseu verkörperte vor-magdalénienzeitliche Kunst und andererseits das Ende des Spätglazials), dass die Hirsche gegen Ende der Sequenz an Bedeutung gewinnen - wie es auch durch die Untersuchung der Reste der Jagdfauna aus den gleichzeitigen Besiedlungsphasen der Höhlen und Abris hinsichtlich der relativen Häufigkeit dieser Art bezeugt ist ${ }^{37}$.

In der älteren Phase handelt es sich im Wesentlichen um Tierdarstellungen im Vollprofil oder im verzerrten Profil, deren vollständige Gliedmaßenpaare ohne Hufe und mittels derselben Konturlinie abgebildet sind. Emmanuel Guy hat, wie oben beschrieben, mehrere konventionelle Formmerkmale bestimmt (geometrische Kontur, einfache Abbildung der Gliedmaßen ohne das Ende der Extremitäten, fehlende Darstellung von Einzelheiten des Körpers), die er als Argument für die Existenz einer künstlerischen Schule betrachtet, diese Schule sei durch gemeinsame Mythogramme gekennzeichnet ${ }^{38}$. Zumeist überlagern sich die Darstellungen aus dieser Zeit traubenartig ${ }^{39}$ und bilden auf diese Weise einen Verbund, der einer geplanten Ordnung unterworfen ist und dessen Linien bis zu einem Viertel der bearbeiteten Oberfläche bedecken ${ }^{40}$.

Die Darstellung der Tierbewegungen geschieht durch segmentale Belebtheit. Diese Belebtheit kann sowohl durch die Abbildung der Momentaufnahme einer Bewegung wie es von einem Großteil der paläolithischen Höhlenkunst bekannt ist ${ }^{41}-$ als auch (vor allem) durch die Aufgliederung der Körpersegmente des Tieres bzw. durch die Darstellung einer Figur mit mehreren Beinen und/oder Köpfen erzeugt werden ${ }^{42}$. Dieses Verfahren, das in der paläolithischen Kunst seltener vorkommt, ist auch in der Höhlenkunst belegt ${ }^{43}$.

In Verbindung mit Tierfiguren, die nach stilistischen Kriterien dem >Mittleren< oder >Oberen< Magdalénien zuzuordnen sind, treten auch karikaturhafte menschliche

37 Zilhão 1997.

38 Guy 2010.

39 Schefer 1999.

40 Guy 2010.

41 Leroi-Gourhan 1992.

42 Luís 2008.

43 Azéma 1992. 
Darstellungen auf. In der jüngsten Phase des Jungpaläolithikums erscheinen geometrische Zeichen und Szenen, in denen mehrere Tiere derselben Art miteinander verknüpft sind.

\subsection{Von welchen natürlichen und menschlichen Faktoren hängt die Verteilung der Petroglyphen im Côa-Tal ab?}

Obgleich die Verbreitungskarte der Felsen mit Petroglyphen das Ergebnis systematischer Begehungen ist, muss das Gesamtbild relativiert werden. Es ist weder möglich denjenigen Teil des Côa-Tales zu begehen, der sich derzeit im Einzugsbereich des Stauwerkes von Pocinho befindet, noch einen großen Teil des Douro-Beckens, dessen portugiesischer Bereich durch mehrere Staudämme in Mitleidenschaft gezogen ist. Ein weiterer unsere Erkenntnismöglichkeiten begrenzender Faktor ist die Verwendung von Bruchstücken der Felsritzungen tragenden Aufschlüsse zur Errichtung von Stützmauern für die landwirtschaftlichen Terrassenkulturen ${ }^{44}$. Es handelt sich dabei um für das Douro-Tal kennzeichnende Anlagen, die seit dem Beginn des 17. Jhs. belegt sind.

Unter Berücksichtigung dieser beiden Faktoren wird gleichwohl deutlich, dass die Bildfelder - ungeachtet ihrer Zeitstellung - keine gleichmäßige Verteilung aufweisen. Vielmehr sind sie auf den letzten acht Kilometern des Tales zusammengedrängt, und zwar genau in dem Bereich, in dem der Wasserlauf die Schiefer der Pinhão-Formation verlässt und in die des Rio Pinhão eintritt. Der Fundort Faia, der von den größeren Bildfeldkonzentrationen relativ isoliert ist, besteht aus zwei Granit-Aufschlüssen, die durch eine kleine Auskragung geschützt sind. In Anbetracht der räumlichen Übereinstimmung mit der Grenze zwischen zwei geologischen Formationen haben wir die Vermutung geäußert, dass in Abhängigkeit von den Auswirkungen klimatischer Faktoren sowie von der Höhenlage, der Temperatur und der mineralischen Zusammensetzung ein unterschiedlicher Erhaltungsgrad der natürlichen Felsoberflächen vorliegt ${ }^{45}$.

Bei den paläolithischen Motiven auf den 330 veröffentlichten Bildfeldern handelt es sich mehrheitlich um Ritzungen, die - auf der Grundlage eines morphologischen und stilistischen Vergleiches mit den Kleinkunst-Stücken aus der stratigraphischen Einheit Nr. 4 vom Fundort Fariseu - der jüngeren Dryaszeit zugerechnet werden können ${ }^{46}$. Diese Bilder befinden sich auf Felsoberflächen, die sich auf alle Hänge verteilen, wobei die größte Dichte im Bereich des Zusammenflusses von Côa und Douro zu verzeichnen ist und kürzlich mehrere neue Bildfelder im Gipfelbereich des bei Vila Nova de Foz Côa gelegenen Plateaus entdeckt worden sind ${ }^{47}$. Demgegenüber drängen sich die gepunzten

44 Baptista - Reis 2008.

45 Aubry et al. 2002.

46 Baptista 2009; Aubry - Sampaio 2008.

47 Baptista - Reis 2008. 
Petroglyphen der älteren Stilphase $4^{8}$ - trotz der systematisch durchgeführten Begehungen - in der unteren Zone zusammen, dabei beschränkt sich ihre Verbreitung im Wesentlichen auf den Bereich der letzten acht Kilometer und die unteren 70 Meter oberhalb der Niedrigwasserlinie.

In einem kürzlich veröffentlichten und durch eine bereits vor einigen Jahren vorgelegte Arbeit ${ }^{49}$ angeregten Aufsat $\mathrm{z}^{50}$ wurde die Vermutung geäußert, dass die Häufung der südlichen und östlichen Expositionsrichtung bei den Bildfeldern, die paläolithische Motive tragen, durch eine anthropogene, zum Zeitpunkt ihrer Herstellung getroffene Wahl bedingt sei (Abb. 2). Die allgemeine Nordwest-Südost Ausrichtung des felsigen Untergrundes entspricht jedoch vollkommen derjenigen, die aus den Übersichten der regionalen tektonischen Verwerfungen hervorgeht, die sich wiederum aus den Auswirkungen der Kompressionsspannung in der jüngsten Phase der variszischen Orogenese ableiten lassen ${ }^{51}$.

Die räumliche Verteilung der der Eisenzeit zuzurechnenden Petroglyphen deckt sich im Übrigen mit derjenigen der aus dem Endpaläolithikum datierenden Darstellungen (Abb. 2). In unterschiedlichen Hanglagen sind mehrfach Überlagerungen von stilistisch dem Spätglazial zuschreibbaren Darstellungen durch eisenzeitliche Motive festzustel$l^{1 e n}{ }^{2}$. Daran zeigt sich, dass die Felsoberflächen zumindest seit dem Ende des Pleistozäns stabil sind. Da die beiden Phasen künstlerischer Darstellung mit ganz verschiedenen Gesellschaften verknüpft sind, ist nur schwer vorstellbar, dass einerseits Jäger und Sammler und andererseits hierarchischen Regeln unterworfene Erzeuger dieselben Bildfelder ausgewählt haben, um darauf sehr unterschiedliche Motive abzubilden.

Vielmehr verhält es sich so, dass die Mehrzahl der der älteren Phase zugehörigen Bildfelder, die sich auf die beiden den Fundorten von Quinta da Barca und Penascosa gegenüberliegenden Hänge verteilen, keine durch jüngere Bilder überlagerten paläolithischen Motive aufweisen (Abb. 9). Diese sind zwar auch an den Fundplätzen vorhanden, befinden sich jedoch auf anderen Bildfeldern ${ }^{53}$. Eine Studie zum Erhaltungsprozess hat gezeigt, dass die Erhaltung der metallisch-siliziösen Patina - bei der es sich um einen der wichtigsten Faktoren für den Schutz der gravierten Felsoberflächen handelt - mit zunehmender Entfernung von der Niedrigwasserlinie des Côa-Flusses schnell abnimmt ${ }^{54}$. Die Unterschiede hinsichtlich der räumlichen Verbreitung der Petroglyphen aus den beiden wichtigsten paläolithischen Kunstphasen - die sich durch einen stilistischen Vergleich anhand der am Fundort Fariseu objektiv gesicherten Daten zeitlich einordnen lassen - sind sowohl durch die Auswahl bestimmter Felsbildträger 55 als auch durch Unterschiede bei den Erhaltungs-

48 Baptista et al. 2008a; Baptista 2008b.

49 Baptista - García Diez 2002.

50 Fernandes 2010.

51 Ribeiro 2001.

52 Zilhão 1997; Baptista - Reis 2008.

53 Baptista - Santos 2010

54 Chauvière et al. 2009.

55 Baptista - Santos 2010. 
bedingungen der Gravierungen der älteren Phase (insbesondere unter dem Einfluss von Temperaturschwankungen während mehrerer Kaltphasen des Spätglazials, die durch dem Felsen Nr. 1 von Fariseu gegenüberliegende Ablagerungen belegt sind) ${ }^{56}$ zu erklären.

Diese verschiedenartigen Feststellungen implizieren, dass die Expositionsrichtung der Bildfelder durch die Ausrichtung des Netzes der tektonischen Frakturen (die entlang der Nordost/Südwest-Achse konzentriert sind) vorgegeben ist. Im Übrigen gehen die Unterschiede im Erhaltungszustand der Gravierungen auf klimatische Faktoren zurück wie auch auf die Verwitterungswirkung, die seit dem Spätglazial durch die Besiedlung mit Flechten und Moosen ausgelöst ist und besonders die nach Nordwesten exponierten Felsoberflächen betrifft57.

Es ist hervorzuheben, dass allein der Felsen Nr. 1 von Fariseu insgesamt 94 von 397 der älteren Phase zuzuordnenden Darstellungen trägt $t^{58}$ und dass das Potential dieses Fundortes, der noch mindestens einen weiteren reich bebilderten Felsen aufweist, nicht ausgeschöpft ist. Diese Feststellung und die Tatsache, dass die beiden Felsen, die die meisten Bildüberlagerungen aufweisen, nahe am Rand der Aufschüttungsebene liegen (Fels Nr. 1 von Quinta da Barca und Fels Nr. 1 von Fariseu), geben uns Anlass zu der Vermutung, dass die Überlieferung der Petroglyphen der älteren Phase in direktem Zusammenhang mit der Existenz einer Abdeckung des Talgrundes durch eine Aufschüttungsebene steht. Die Entdeckung des Felsens Nr. 1 von Fariseu zeigt, dass folglich noch zahlreiche Felsbildträger unter den Anschwemmungen zu entdecken sein werden ${ }^{59}$.

\subsection{Von wo aus sind die Bilder sichtbar und in welcher Weise stehen sie zueinander in Beziehung?}

Die Sichtbarkeit ist nach den für die paläolithischen Bilder des Côa-Tales vorliegenden funktionalen Deutungen ein bedeutender Faktor ${ }^{60}$. Gleichwohl weisen die Positionsmerkmale der Gravierungen mit Bezug auf ihre natürliche Umgebung in den beiden zeichnerischen Hauptphasen des Paläolithikums deutliche Unterschiede auf.

In der älteren Phase treten die Bildfelder hauptsächlich im Randbereich der Aufschüttungsebene des Côa-Flusses auf, so dass mehrfach auf ihre Beziehung zum Wasserlauf bzw. seine jahreszeitlich bedingten Schwankungen hingewiesen wurde ${ }^{61}$. In den ergiebigsten Bereichen sind die Bildfelder häufig wie auf einem Podest angeordnet. Diese Gliederung ergibt sich aus dem Zusammenspiel der Ausrichtung der Schieferlagen des Muttergesteins und der parallelen Brüche, die das Muttergestein durchziehen (Abb. 5). Diese

56 Aubry et al. 2010.

57 Aubry et al. 2012.

58 Baptista et al. 2008a; Baptista et al. 2008b.

59 Aubry et al. 2010.

60 Baptista - García Diez 2002.

61 Schefer 1999. 
Häufungen [Anm. d. Übers.: von Bildfeldern] befinden sich am Rand der Hanglagen ${ }^{62}$ in den Bereichen, in denen das Tal breiter wird und sich derzeit Anschwemmungen ablagern. Isolierte Felsen mit einer geringeren Zahl von Bildern stehen in höheren Hanglagen oder entlang der zeitweiligen Zuflüsse des Côa-Flusses. Die Anhäufungen nebeneinander gesetzter Bilder auf ein und demselben Bildträger wurden dahingehend gedeutet, dass eine Monumentalisierung des natürlichen Raumes beabsichtigt gewesen sein könnte ${ }^{63}$.

Innerhalb dieser absichtlichen Störung durch die Überlagerung von Bildern - die jeweils vereinfachte taxonomische Merkmale aufweisen und unmittelbar lesbar sind wird von einigen Autoren ${ }^{64}$ für die dieser Phase der Fundorte Quinta da Barca, Penascosa, Fariseu und Canada do Inferno zugeschriebenen Bilder die Existenz struktureller Muster angenommen, bei denen die dargestellten Arten, die Größe und Ausrichtung der Figuren sowie der Abstand zwischen den Felsen eine Rolle spielten. Die jeweiligen Anteile der auf den einzelnen Bildfeldern dargestellten Tierarten hätten darüber hinaus unterschiedliche Bereiche innerhalb dieser Felsbildfundorte definiert (hauptsächlich Hirsch, hauptsächlich Aurochse, usw.). Durch die Orientierung der jeweiligen Figurenmehrheit auf einem Bildfeld schließlich sei auch eine Laufrichtung im Rahmen eines die verschiedenen Bildfelder miteinander verbindenden Parcours definiert worden.

Die Bilder aus der Endphase des Paläolithikums sind demgegenüber von geringerer Größe, sie weisen nur selten Überlagerungen auf und sind in den Fällen, in denen mehrere Tiere derselben Art miteinander verknüpft sind, szenisch angeordnet. Sie befinden sich auf Bildfeldern, die sich über die gesamten Hanglagen verteilen. Im Hinblick auf die Bildfelder, die Darstellungen dieser Periode aufweisen, wurde die Vermutung geäußert, dass sie zwar nach wie vor dieselbe Funktion hatten, ihre sequentielle Visualisierung jedoch die Anwesenheit eines über ihr Vorhandensein informierten >Führers< erfordert hätte, was mehrere Erkenntnisebenen implizieren würde ${ }^{65}$.

Um diese Beobachtungen zur Sichtbarkeit der Darstellungen in den beiden wichtigsten zeichnerischen Phasen genauer zu bestimmen, wurde nach Techniken untergliedert der den Bildern zugeschriebene Sichtbarkeitsabstand in messbare Größen umgesetzt. Aufgrund der Untersuchungsergebnisse schlagen wir eine Deutung der paläolithischen Darstellungen vor.

Es wurde eine Versuchsreihe mit der Zielsetzung durchgeführt, die Sichtbarkeit der Linien auf einem - nach der Farbskala der Bodenfarben von André Cailleux ${ }^{66}$ - dunkelbraunen Bilduntergrund (Farbton R-69) zu bewerten. Die mit Hilfe von zwei Personen die sich auf ebenem Untergrund schrittweise von der Felswand entfernten - erarbeiteten Ergebnisse erlauben eine Quantifizierung der maximalen Entfernung, bei der übereinstimmend breite Linien in drei unterschiedlichen Farbtönen noch erkennbar sind (Abb. 8).

62 Baptista - García Diez 2002.

63 Zilhão 1998.

64 Baptista et al. 2006; Baptista et al. 2008a; Baptista et al. 2008b; Baptista - Santos 2010.

65 Baptista - Santos 2010.

66 Cailleux 1979. 


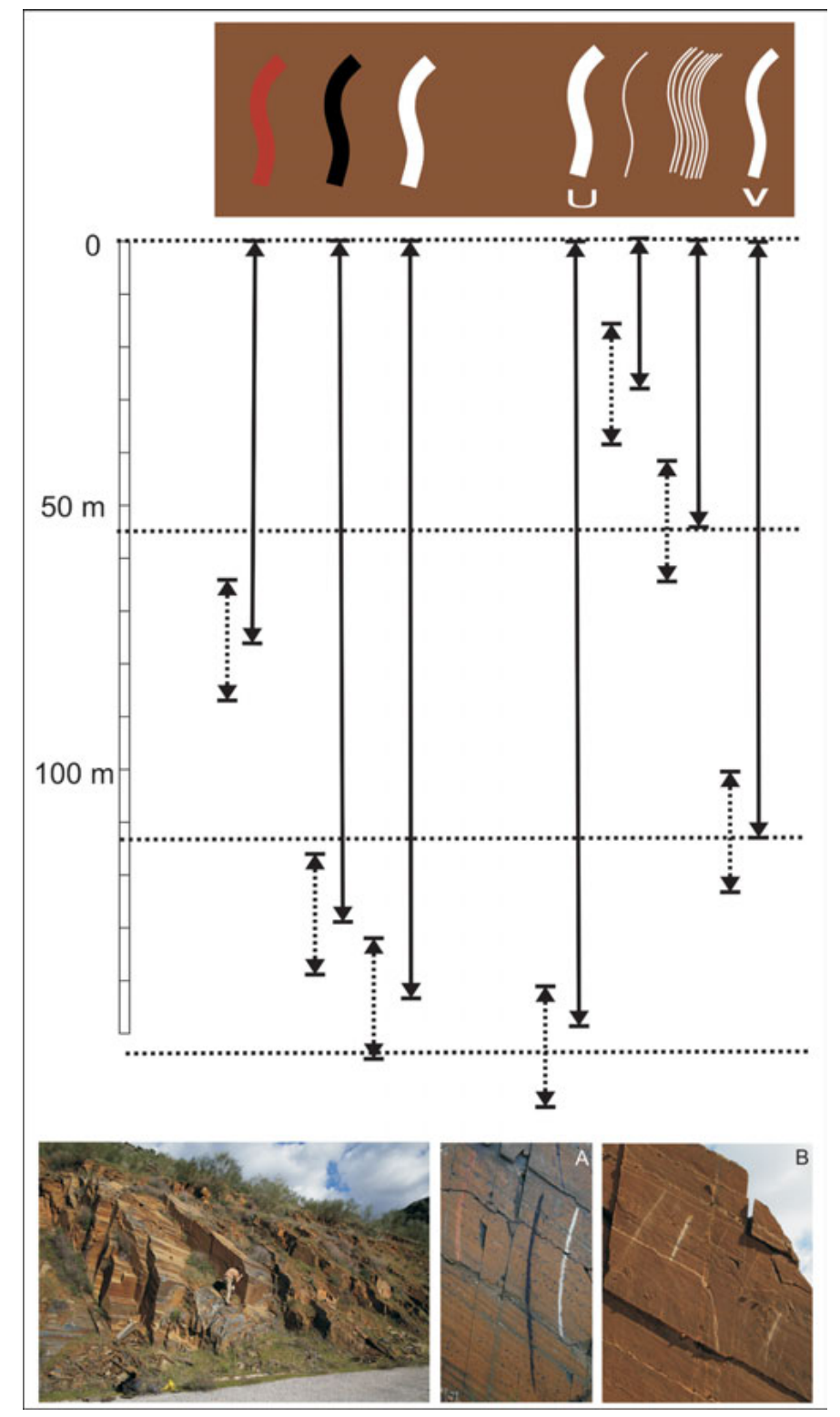

Abb. 8 | Experimentelles Bezugssystem zur Bewertung der Sichtbarkeit anhand von Farbe und verwendeter Technik. 


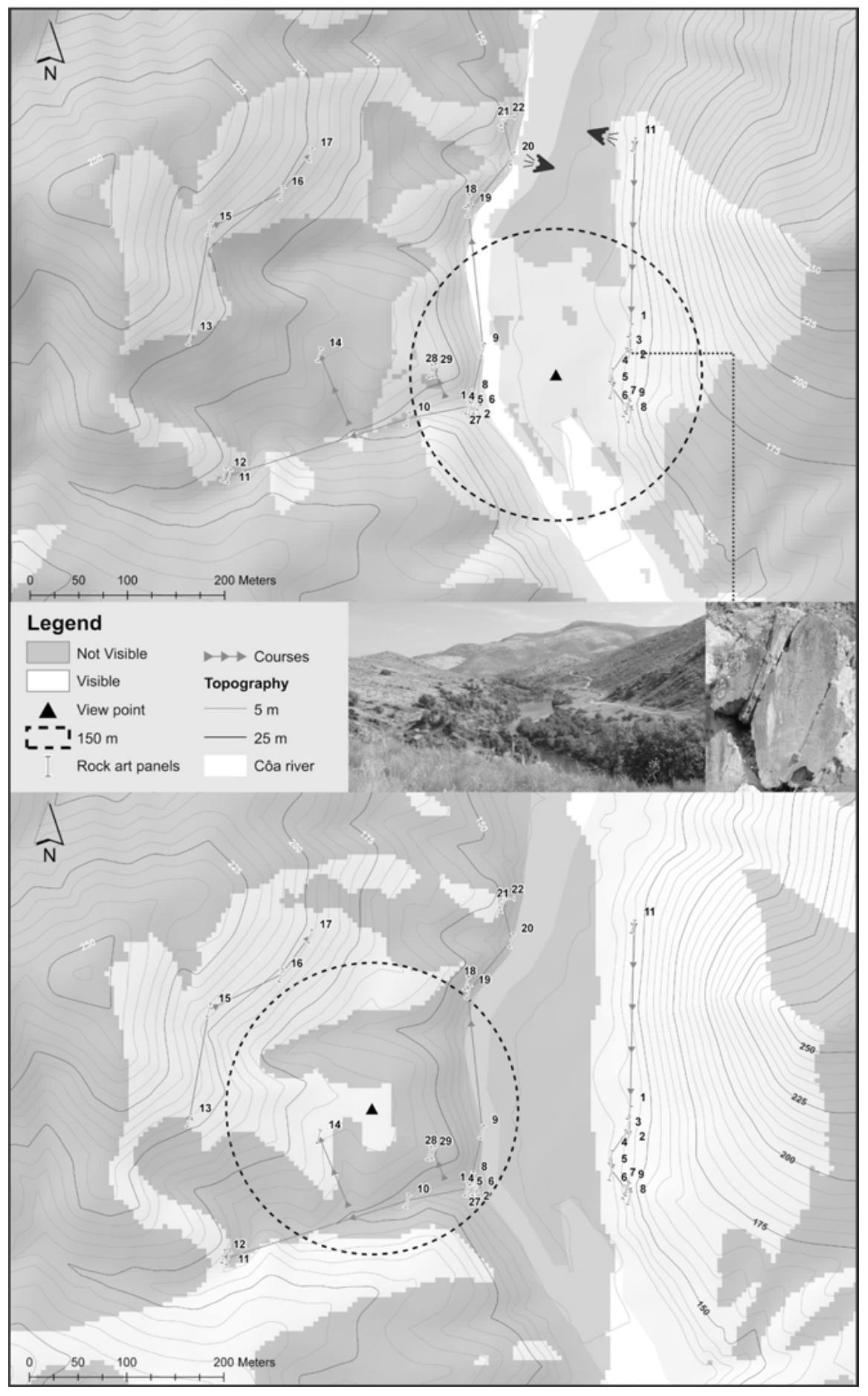

Abb. 9 | Sichtbarkeit der Dar stellungen der älteren Phase an den Fundorten Quinta da Barca und Penscosa (M. $1: 7500)$. 

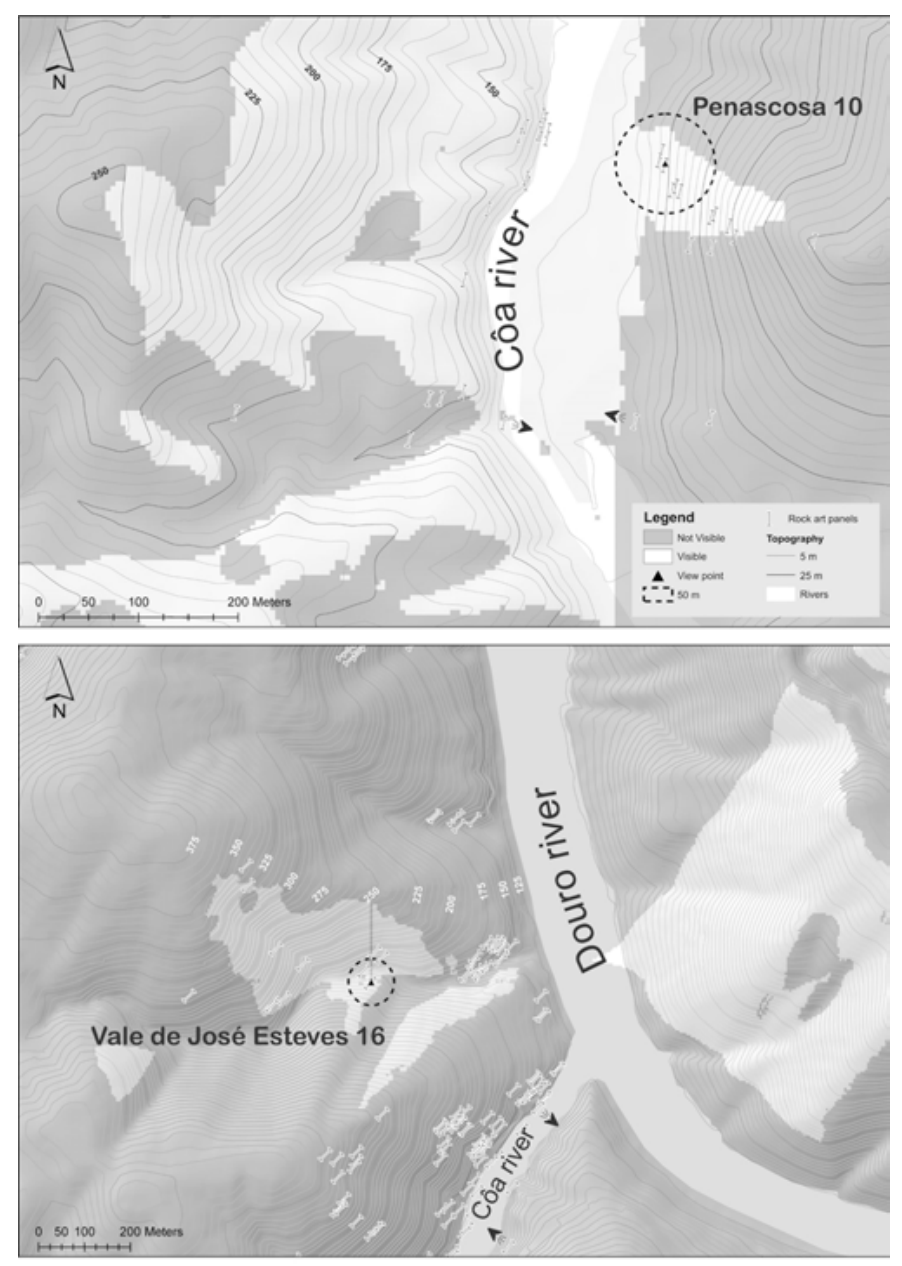

Abb. 10 | Sichtbarkeit der vom Ende des Jungpaläolithikums datierenden Darstellungen auf Felsen der Fundorte Vale de José Esteves, Vermelhosa und Penascosa.

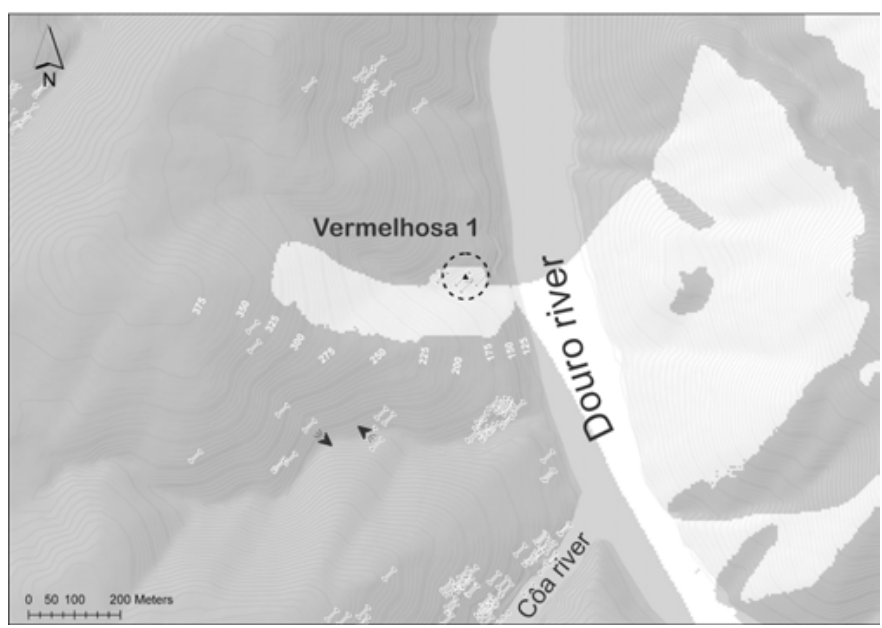




\begin{tabular}{|c|c|c|c|c|c|c|c|c|c|c|c|c|c|c|c|c|c|c|c|c|c|c|c|c|c|c|}
\hline & & & \multicolumn{23}{|c|}{ Observed } & \multirow{3}{*}{$\sqrt{e^{2}}$} \\
\hline & & & \multicolumn{9}{|c|}{ Course I } & \multicolumn{2}{|c|}{$\mathrm{IA}$} & IB & \multicolumn{7}{|c|}{ Course II } & \multicolumn{4}{|c|}{ Course III } & \\
\hline & & $\mathrm{R}$ & 6 & 1 & 27 & 2 & 4 & 5 & 10 & 11 & 12 & 28 & 29 & 14 & 8 & 9 & 18 & 19 & 20 & 21 & 22 & 13 & 15 & 16 & 17 & \\
\hline \multirow{26}{*}{ 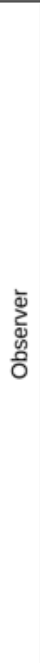 } & \multirow{9}{*}{ 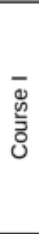 } & 6 & & 口 & 4 & 4 & 4 & 4 & 0 & 0 & 0 & 0 & 0 & 0 & 4 & 0 & 0 & 0 & 0 & 1 & 1 & 0 & 0 & 0 & 0 & 26 \\
\hline & & 1 & 2 & & $\mathrm{Q}$ & 4 & 4 & 4 & 0 & 0 & 0 & 0 & 0 & 0 & 4 & 0 & 0 & 0 & 0 & 1 & 1 & 0 & 0 & 0 & 0 & 24 \\
\hline & & 27 & 2 & 2 & & $\square$ & 4 & 4 & 0 & 0 & 0 & 0 & 0 & 0 & 2 & 0 & 0 & 0 & 0 & 1 & 1 & 0 & 0 & 0 & 0 & 20 \\
\hline & & 2 & 2 & 2 & 2 & & 口 & 4 & 0 & 0 & 0 & 0 & 0 & 0 & 2 & 0 & 0 & 0 & 0 & 1 & 1 & 0 & 0 & 0 & 0 & 18 \\
\hline & & 4 & 2 & 2 & 2 & 2 & & 口 & 0 & 0 & 0 & 0 & 0 & 0 & 2 & 0 & 0 & 0 & 0 & 0 & 0 & 0 & 0 & 0 & 0 & 14 \\
\hline & & 5 & 2 & 2 & 2 & 2 & 2 & & 口 & 0 & 0 & 0 & 0 & 0 & 2 & 0 & 0 & 0 & 0 & 0 & 0 & 0 & 0 & 0 & 0 & 12 \\
\hline & & 10 & 0 & 0 & 0 & 0 & 0 & 0 & & 口 & 0 & 0 & 0 & 0 & 0 & 0 & 0 & 0 & 0 & 0 & 0 & 0 & 0 & 0 & 0 & 0 \\
\hline & & 11 & 0 & 0 & 0 & 0 & 0 & 0 & 0 & & 口 & 0 & 0 & 0 & 0 & 0 & 0 & 0 & 0 & 0 & 0 & 0 & 0 & 0 & 0 & 4 \\
\hline & & 12 & 0 & 0 & 0 & 0 & 0 & 0 & 0 & 2 & & 0 & 0 & 0 & 0 & 0 & 0 & 0 & 0 & 0 & 0 & 0 & 0 & 0 & 0 & 2 \\
\hline & \multirow{2}{*}{$\leq$} & 28 & 0 & 0 & 0 & 0 & 0 & 0 & 4 & 3 & 3 & \multicolumn{2}{|r|}{$\square$} & 0 & 0 & 0 & 0 & $\bar{c}$ & 0 & 2 & 2 & 0 & 3 & 3 & 3 & 27 \\
\hline & & 29 & 0 & 0 & 0 & 0 & 0 & 0 & 4 & 3 & 3 & \multicolumn{2}{|c|}{2} & 0 & 0 & 0 & 0 & 0 & 0 & 2 & 2 & 0 & 3 & 3 & 3 & 25 \\
\hline & $\underline{\underline{m}}$ & 14 & 0 & 0 & 0 & 0 & 0 & 0 & 0 & 0 & 0 & 0 & 0 & & 0 & 0 & 0 & 0 & 0 & 0 & 0 & 4 & 3 & 3 & 3 & 13 \\
\hline & \multirow{7}{*}{ 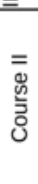 } & 8 & 2 & 2 & 2 & 2 & 2 & 4 & 0 & 0 & 0 & 0 & 0 & 0 & \multicolumn{2}{|r|}{$\bar{D}$} & \multirow{2}{*}{$\begin{array}{l}0 \\
\square\end{array}$} & 0 & 0 & 0 & 1 & 0 & 0 & 0 & 0 & 15 \\
\hline & & 9 & 0 & 0 & 0 & 0 & 0 & 0 & 0 & 0 & 0 & 0 & 0 & 0 & 0 & & & 2 & 0 & 1 & 1 & 0 & 0 & 0 & 0 & 5 \\
\hline & & 18 & 0 & 0 & 0 & 0 & 0 & 0 & 0 & 0 & 0 & 0 & 0 & 0 & 0 & 1 & \multicolumn{2}{|c|}{$\square$} & \multirow{2}{*}{$\square^{0}$} & 0 & 0 & 0 & 0 & 0 & 0 & 1 \\
\hline & & 19 & 0 & 0 & 0 & 0 & 0 & 0 & 0 & 0 & 0 & 0 & 0 & 0 & 0 & 2 & 4 & & & 0 & 0 & 0 & 0 & 0 & 0 & 6 \\
\hline & & 20 & 0 & 0 & 0 & 0 & 0 & 0 & 0 & 0 & 0 & 0 & 0 & 0 & 0 & 0 & 0 & 0 & & 口 & 4 & 0 & 0 & 0 & 0 & 8 \\
\hline & & 21 & 0 & 0 & 0 & 0 & 0 & 0 & 0 & 0 & 0 & 0 & 0 & 0 & 0 & 1 & 0 & 0 & 2 & & $\mathrm{Q}$ & 0 & 0 & 0 & 0 & 7 \\
\hline & & 22 & 0 & 0 & 0 & 0 & 0 & 0 & 0 & 0 & 0 & 0 & 0 & 0 & 0 & 1 & 0 & 0 & 2 & 2 & & 口 & 0 & 0 & 0 & 5 \\
\hline & $\equiv$ & 13 & 0 & 0 & 0 & 0 & 0 & 0 & 0 & $\overline{0}$ & 0 & 0 & 0 & 0 & 0 & 0 & 0 & 0 & 0 & 0 & 0 & & $\square$ & 0 & 0 & 0 \\
\hline & ષ્ఝ & 15 & 0 & 0 & 0 & 0 & 0 & 0 & 0 & 0 & 0 & 0 & 0 & 1 & 0 & 0 & 0 & 0 & 0 & 0 & 0] & 0 & & 口 & 0 & 1 \\
\hline & 比 & 16 & 0 & 0 & 0 & 0 & 0 & 0 & 0 & 0 & 0 & 0 & 0 & 1 & 0 & 0 & 0 & 0 & 0 & 0 & 0 & 0 & 0 & & 口 & 1 \\
\hline & ن & 17 & 0 & 0 & 0 & 0 & 0 & 0 & 0 & 0 & 0 & 0 & 0 & 1 & 0 & 0 & 0 & 0 & 0 & 0 & 0 & 0 & 0 & 0 & & 1 \\
\hline & Inc & & 12 & 14 & 16 & 18 & 20 & 24 & 8 & 8 & 10 & 2 & 4 & 3 & 16 & 5 & 5 & 2 & 4 & 15 & 18 & 4 & 9 & 9 & 9 & \\
\hline & & $\mathrm{A}$ & 4 & 4 & 4 & 4 & 4 & 4 & 0 & 0 & 3 & 4 & 4 & 0 & 4 & 4 & 3 & 3 & 3 & 3 & 3 & 0 & 3 & 3 & 3 & 67 \\
\hline & & B & 0 & 0 & 0 & 0 & 0 & 0 & 0 & 0 & 3 & 0 & 0 & 4 & 0 & 0 & 0 & 0 & 0 & 2 & 0 & 3 & 3 & 3 & 3 & 21 \\
\hline
\end{tabular}

Tab. 1 | Sichtbarkeit zwischen den Bildfeldern von Quinta da Barca. Die Abfolge zwischen den Bildfeldern folgt der von Baptista et al. 2008b vorgeschlagenen Anordnung. Die Werte entsprechen: nicht sichtbar (o), Sichtbarkeit des Aufschlusses außerhalb des Lesbarkeitsbereiches der Linien von $150 \mathrm{~m}$ (1), Sichtbarkeit des Aufschlusses innerhalb des Lesbarkeitsbereiches der Linien (2), Sichtbarkeit der Bildfeldoberfläche außerhalb des Lesbarkeitsbereiches der Linien (3) und Sichtbarkeit der Bildfeldoberfläche und der Linien innerhalb des Lesbarkeitsbereiches (4).

Bezüglich der Oberflächen, die die im Tal am häufigsten vertretenen Farbtöne aufweisen, war festzustellen, dass die gepunzte Linie, die darauf einen weißen Kontrast bildet, über die größte räumliche Distanz hinweg sichtbar ist. In einem zweiten Schritt wurden unter Einsatz mehrerer im Paläolithikum verwendeter Techniken Felsbildlinien erzeugt und die maximale Sichtbarkeitsentfernung quantifiziert. Schließlich wurde ein Versuch durchgeführt, bei dem die Beobachter sich schrittweise den in den verschiedenen Techniken als Zahlen ausgeführten Darstellungen näherten.

Es ist nun möglich, bezüglich der in den unterschiedlichen Techniken ausgeführten frischen Linien, die den größten Kontrast gegenüber dem Untergrund aufweisen, einen Grenzwert für die Sichtbarkeit der gravierten Darstellungen anzugeben. Wir haben diesen Ansatz auf Bildfelder der beiden Kunstphasen des Jungpaläolithikums angewendet: Dies betraf an den Fundorten Penascosa/Quinta da Barca die ältere Phase (Abb. 9) und auf einem Felsen von Vale de José Esteves, Vermelhosa und Penascosa das Ende des Jungpaläolithikums (Abb. 10)

Die im Rahmen der Versuchsreihen gewonnenen Daten wurden unter Nutzung der Sichtbarkeitsanalyse in einem geographischen Informationssystem auf die während des 


\begin{tabular}{|c|c|c|c|c|c|c|c|c|c|c|c|c|c|c|c|c|c|c|c|c|c|c|c|c|c|c|}
\hline & & \multicolumn{23}{|c|}{ Observed } & \multirow[b]{3}{*}{ Index } \\
\hline & & & \multicolumn{23}{|c|}{ Quinta da Barca } & \\
\hline & & $\mathrm{R}$ & 6 & 1 & 27 & 2 & 4 & 5 & 10 & 11 & 12 & 28 & 29 & 14 & 8 & 9 & 181 & 19 & 20 & 21 & 22 & 13 & 15 & 16 & 17 & \\
\hline \multirow{10}{*}{ 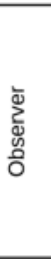 } & \multirow{10}{*}{ 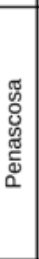 } & 11 & 3 & 3 & 3 & 3 & 3 & 3 & 0 & 0 & 0 & 0 & 0 & 0 & 3 & 3 & 3 & 3 & 4 & 4 & 2 & 0 & 3 & 3 & 3 & 49 \\
\hline & & 1 & 3 & 3 & 3 & 3 & 3 & 3 & 0 & 3 & 3 & 3 & 3 & 0 & 3 & 3 & 3 & 3 & 3 & 3 & 3 & 3 & 3 & 3 & 3 & 63 \\
\hline & & 2 & 3 & 3 & 3 & 3 & 3 & 3 & 0 & 0 & 3 & 3 & 3 & 0 & 3 & 3 & 3 & 3 & 3 & 3 & 3 & 3 & 3 & 3 & 3 & 60 \\
\hline & & 3 & 3 & 3 & 3 & 3 & 3 & 3 & 0 & 0 & 3 & 3 & 3 & 0 & 3 & 4 & 3 & 3 & 3 & 3 & 3 & 3 & 3 & 3 & 3 & 61 \\
\hline & & 4 & 4 & 4 & 4 & 4 & 3 & 3 & 0 & 0 & 3 & 3 & 3 & 0 & 4 & 4 & 3 & 3 & 3 & 3 & 3 & 3 & 3 & 3 & 3 & 66 \\
\hline & & 5 & 4 & 4 & 4 & 4 & 4 & 4 & $\mathbf{0}$ & $\mathbf{0}$ & 3 & 3 & 3 & 0 & 4 & 4 & 3 & 3 & 3 & 3 & 3 & 3 & 3 & 3 & 3 & 68 \\
\hline & & 6 & 3 & 3 & 3 & 3 & 3 & 3 & 0 & 0 & 0 & 3 & 3 & 0 & 3 & 3 & 3 & 3 & 3 & 3 & 3 & 3 & 3 & 3 & 3 & 57 \\
\hline & & 7 & 3 & 3 & 3 & 3 & 3 & 3 & 0 & 0 & 3 & 3 & 3 & 0 & 3 & 3 & 3 & 3 & 3 & 3 & 3 & 3 & 3 & 3 & 3 & 60 \\
\hline & & 9 & 3 & 3 & 3 & 3 & 3 & 3 & 0 & 0 & 3 & 3 & 3 & 0 & 3 & 3 & 3 & 3 & 3 & 3 & 3 & 3 & 3 & 3 & 3 & 60 \\
\hline & & 8 & 3 & 3 & 3 & 3 & 3 & 3 & 0 & 0 & 3 & 3 & 3 & 0 & 3 & 3 & 3 & 3 & 3 & 3 & 3 & 3 & 3 & 3 & 3 & 60 \\
\hline \multicolumn{3}{|c|}{ Index } & 32 & 32 & 32 & 32 & 31 & 31 & 0 & 3 & 24 & 27 & 27 & 0 & 32 & 33 & 30 & 30 & 31 & 31 & 29 & 27 & 30 & 30 & 30 & \\
\hline
\end{tabular}

Tab. 2 | Sichtbarkeit der Bildfelder von Penascosa in Quinta da Barca (s. Tab. 1 für weitere Einzelheiten).

\begin{tabular}{|c|c|c|c|c|c|c|c|c|c|c|c|c|}
\hline & & \multicolumn{10}{|c|}{ Observed } & 㐅㐅 \\
\hline & $\mathrm{R}$ & 11 & 1 & 2 & 3 & 4 & 5 & 6 & 7 & 9 & 8 & \\
\hline \multirow{13}{*}{ ठิ } & 11 & & 口 & 0 & 0 & 0 & 0 & 0 & 0 & 0 & 0 & 0 \\
\hline & 1 & 0 & & 口 & 2 & 2 & 0 & 0 & 0 & 0 & 0 & 6 \\
\hline & 2 & 0 & 2 & & 口 & 2 & 0 & 0 & 0 & 0 & 0 & 6 \\
\hline & 3 & 0 & 2 & 4 & & 口 & 0 & 0 & 0 & 0 & 0 & 8 \\
\hline & 4 & 0 & 4 & 4 & 4 & & 口 & 4 & 4 & 4 & 0 & 26 \\
\hline & 5 & 0 & 4 & 0 & 0 & 2 & & 口 & 4 & 4 & 0 & 18 \\
\hline & 6 & 0 & 2 & 0 & 0 & 2 & 2 & & 口 & 4 & 4 & 18 \\
\hline & 7 & 0 & 0 & 0 & 0 & 2 & 2 & 2 & & 口 & 0 & 10 \\
\hline & 9 & 0 & 2 & 2 & 2 & 2 & 2 & 2 & 2 & & 口 & 14 \\
\hline & 8 & 0 & 0 & 0 & 0 & 2 & 2 & 2 & 0 & 0 & & \\
\hline & Inde & 0 & 16 & 12 & 10 & 16 & 10 & 14 & 14 & 16 & 4 & \\
\hline & A & 1 & 4 & 4 & 4 & 4 & 4 & 4 & 4 & 4 & 4 & 37 \\
\hline & B & 3 & 3 & 3 & 3 & 3 & 3 & 3 & 3 & 3 & 3 & 30 \\
\hline
\end{tabular}

Tab. 3 | Sichtbarkeit zwischen den Bildfeldern von Penascosa (s. Tab. 1 für weitere Einzelheiten).

Tab. 4 | Sichtbarkeit der Bildfelder von Quinta da Barca in Penascosa (s. Tab. 1 für weitere Einzelheiten).

\begin{tabular}{|c|c|c|c|c|c|c|c|c|c|c|c|c|}
\hline & & \multicolumn{10}{|c|}{$\begin{array}{c}\text { Observed } \\
\text { Penascosa }\end{array}$} & \multirow{2}{*}{ 希 } \\
\hline & $\mathrm{R}$ & 11 & 1 & 2 & 3 & 4 & 5 & 6 & 7 & 9 & 8 & \\
\hline \multirow{23}{*}{ 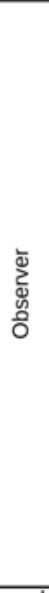 } & 6 & 3 & 3 & 3 & 3 & 4 & 4 & 3 & 3 & 3 & 3 & 32 \\
\hline & 1 & 3 & 3 & 3 & 3 & 4 & 4 & 3 & 3 & 3 & 3 & 32 \\
\hline & 27 & 3 & 3 & 3 & 3 & 4 & 4 & 3 & 3 & 3 & 3 & 32 \\
\hline & 2 & 3 & 3 & 3 & 3 & 4 & 4 & 3 & 3 & 3 & 3 & 32 \\
\hline & 4 & 3 & 3 & 3 & 3 & 4 & 4 & 3 & 3 & 3 & 3 & 32 \\
\hline & 5 & 3 & 3 & 3 & 3 & 3 & 4 & 3 & 3 & 3 & 3 & 31 \\
\hline & 10 & 0 & 3 & 3 & 3 & 3 & 3 & 3 & 3 & 3 & 3 & 27 \\
\hline & 11 & 0 & 3 & 0 & 0 & 0 & 0 & 0 & 0 & 0 & 0 & 3 \\
\hline & 12 & 0 & 3 & 0 & 0 & 0 & 0 & 0 & 0 & 0 & 0 & \\
\hline & 28 & 3 & 3 & 3 & 3 & 3 & 3 & 3 & 3 & 3 & 3 & 30 \\
\hline & 29 & 3 & 3 & 3 & 3 & 3 & 3 & 3 & 3 & 3 & 3 & 30 \\
\hline & 14 & 0 & 0 & 0 & 0 & 0 & 0 & 0 & 0 & 0 & 0 & 0 \\
\hline & 8 & 3 & 3 & 3 & 3 & 4 & 4 & 3 & 3 & 3 & 3 & 32 \\
\hline & 9 & 3 & 3 & 3 & 4 & 4 & 4 & 3 & 3 & 3 & 3 & 33 \\
\hline & 18 & 3 & 3 & 3 & 3 & 3 & 3 & 3 & 3 & 3 & 3 & 30 \\
\hline & 19 & 3 & 3 & 3 & 3 & 3 & 3 & 3 & 3 & 3 & 3 & 30 \\
\hline & 20 & 4 & 3 & 3 & 3 & 3 & 3 & 3 & 3 & 3 & 3 & 31 \\
\hline & 21 & 4 & 3 & 3 & 3 & 3 & 3 & 3 & 3 & 3 & 3 & 31 \\
\hline & 22 & 4 & 3 & 3 & 3 & 3 & 3 & 3 & 3 & 3 & 3 & 31 \\
\hline & 13 & 3 & 3 & 3 & 3 & 3 & 3 & 3 & 3 & 3 & 3 & 30 \\
\hline & 15 & 3 & 3 & 3 & 3 & 3 & 3 & 3 & 3 & 3 & 3 & 30 \\
\hline & 16 & 3 & 3 & 3 & 3 & 3 & 3 & 3 & 3 & 3 & 3 & 30 \\
\hline & 17 & 3 & 3 & 3 & 3 & 3 & 3 & 3 & 3 & 3 & 3 & 30 \\
\hline Inder & & 60 & 66 & 60 & 61 & 67 & 73 & 60 & 60 & 60 & 60 & \\
\hline
\end{tabular}

Paläolithikums gearbeiteten Bildfelder angewendet ${ }^{67}$. Die verschiedenen Bildfelder und die Darstellungen der älteren Phase an den Fundorten Penascosa und Quinta da Barca wurden insgesamt auf die Sichtbarkeit der Linien, der Oberflächen und des Aufschlusses hin betrachtet. Darüber hinaus wurde die durch die bevorzugte Ausrichtung der Klüfte (NNO/SSW in Penascosa und Quinta da Barca, NO/SW an den übrigen Fundorten) vorgegebene Ausrichtung der Bildfelder einbezogen (Tab. 1-4). Im Hinblick auf die Vermutung, dass sie ein und demselben Parcours zugehören, zeigen die Ergebnisse, dass sich die vorgeschlagenen Strecken nicht vollständig durch die Ortung der entlegenen Darstellungen erklären lassen ${ }^{68}$. In zahlreichen Fällen sind sie, aufgrund der Entfernung und der Topographie des dazwischen liegenden Geländes, nicht erkennbar.

67 Wheatley - Gillings 2002.

68 Baptista et al. 2008b; Baptista - Santos 2010. 
Unter der Voraussetzung, dass eine stilistische Homogenität einem übereinstimmenden symbolischen Code entspricht, lassen sich mit Rücksicht auf die örtliche Topographie anhand der räumlichen Analyse alternativ diejenigen Plätze bestimmen, von denen aus die größte Zahl von Bildfeldern lesbar ist. Der Punkt A befindet sich [Anm. d. Übers.: mit Bezug auf den Parcours] auf halbem Wege von der Aufschüttungsebene entfernt (Abb. 9). Von dort aus sind die Linien auf allen Bildfeldern des Fundortes sichtbar - mit Ausnahme des Felsens Nr. 11, der in einer Entfernung von 253 Metern flussabwärts liegt. Darüber hinaus ist von dort aus der größte Teil der Gravierungen sichtbar, die sich am Hangfuß des Fundplatzes Quinta da Barca befinden. Der Punkt B liegt auf einer durch eine Aufschüttungsterrasse gebildeten Plattform. Von dort aus sind die Bildfelder im oberen Hangbereich des Fundortes Quinta da Barca sowie die am Rand der Aufschüttungsebene von Penascosa befindlichen Bildfelder sichtbar, wobei jedoch die gravierten Linien nicht erkennbar sind.

Auf die an das Ende des Jungpaläolithikums datierte jüngere zeichnerische Phase bezogen ist vor dem Hintergrund der Versuchsergebnisse festzustellen, dass sich - im Unterschied zu den gepunzten Gravierungen der älteren Phase - die Sichtbarkeit ihrer Bilder auf den Raum der kleinen Plattformen beschränkt, die direkt an den Bildfeldern liegen (Abb. 10). Von keinem Punkt aus sind die Darstellungen von mehr als zwei verschiedenen Bildfeldern lesbar (Vale do José Esteves 16, Vermelhosa 1, Penascosa 10).

\section{3 Übertragung der aus Côa bekannten Gegebenheiten auf einen größeren Maßstab}

Trotz fehlender systematischer Begehungen im größten Teil Portugals und Spaniens und der Schwierigkeiten, die mit der Auffindung patinierter, fein eingeritzter Gravierungen verbunden sind, zeigt die verfügbare Verbreitungskarte ${ }^{69}$, dass die im unteren Côa-Tal vorhandene Dichte künstlerischer Zeugnisse des Paläolithikums und der Eisenzeit entlang der nahe gelegenen Nebenflüsse des Douro, die noch nicht durch Staudammbauten (Ribeira de Aguiar, Àgueda) in Mitleidenschaft gezogen worden sind, keine Entsprechung hat. Im oberen Bereich dieses Einzugsgebietes und desjenigen weiterer Nebenflüsse des Douro dürfte jedoch während des letzten glazialen Maximums ein ähnliches durch Regen und Schnee gekennzeichnetes Niederschlagsregime vorgeherrscht haben, wie wir es für das Côa-Tal rekonstruiert haben. Bei letzterem ist dies eine mögliche Erklärung für dessen Besiedlung während des Jungpaläolithikums ${ }^{70}$. Am Fundort Siega Verda können nur einige wenige Darstellungen unter stilistischen Gesichtspunkten mit den Bildern der älteren Phase des Côa-Tales gleichgesetzt werden ${ }^{71}$. Die in einem aus dem jüngeren

69 Baptista 2009; Reis mündliche Mitteilung.

70 Aubry et al. 2002.

71 Guy 2010. 

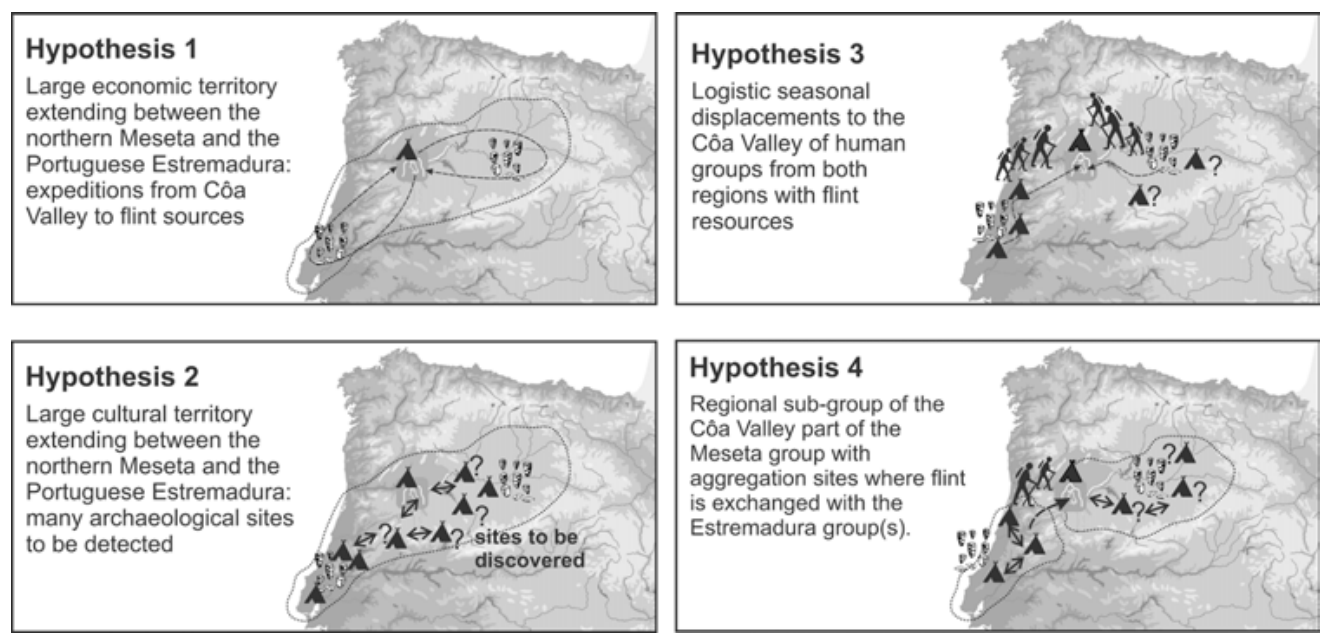

Abb. 1 | Herkunft der während des Jungpaläolithikums an den Fundstellen des Côa-Tales verwendeten Silexstücke und mögliche Deutungsmodelle.

Holozän datierenden, alluvialen sedimentären Zusammenhang erhaltenen Felsbilder ${ }^{22}$ könnten ein Gegenstück zu den in Penascosa und Quinta da Barca entdeckten Petroglyphen darstellen. Sie könnten darüber hinaus als Hinweis auf die Existenz weiterer, unter Sedimenten verborgener Fundplätze im flussabwärts gelegenen Teil des Einzugsgebietes zu werten sein.

Hinsichtlich der für das Coâ-Tal charakteristischen Ausdehnung und großen Dichte der Felsbildfundplätze sind mehrere, auch einander ergänzende Erklärungen möglich. Eine erste Vermutung besagt, dass die Überlieferung der Gravierung das Ergebnis des Zusammenspiels natürlicher Faktoren sei, die mit der Art des geologischen Untergrundes, der Tektonik und den während des Pleistozäns und des Holozäns herrschenden klimatischen Bedingungen zusammenhängen würden und bessere Erhaltungsbedingungen als anderswo geboten hätten. Auch ist vorstellbar, dass sich - abgesehen von den besonderen Erhaltungsbedingungen - die Dichte der erhaltenen Gravierungen aus einer im Vergleich zu den angrenzenden Regionen intensiveren Besiedlung des Tales aufgrund besonderer Merkmale und Ressourcen ergibt. Wobei dies sowohl für die Jäger- und Sammlergesellschaften als auch für die Bauern und Viehzüchter gilt.

Die Untersuchung der Herkunft der an den Fundorten des Côa-Tales - einer Region, in der dieser Rohstoff nicht natürlich vorkommt - zurückgelassenen Silexstücke liefert weitere Informationen, die das Bild der Verbreitung der paläolithischen Felsbilder und der sonstigen Fundstellen abrunden. Auf dieser Grundlage ist nicht nur eine Bewertung der 
Größe der genutzten Territorien möglich, sondern es können auch mehrere Hypothesen bezüglich des zugrundeliegenden sozialen Netzwerkes entwickelt werden, die zur Erklärung derartiger Bewegungen beitragen ${ }^{73}$ (Abb. 11). Als Ergebnis dieser Untersuchung ist festzuhalten, dass Silex aus zwei einander entgegengesetzt liegenden Regionen verwendet wurde - das Material stammte zum einen aus der im Osten gelegenen Meseta und zum anderen aus Quellen, die sich im Südwesten, im Sedimentgestein Zentralportugals befanden. Dabei ist hervorzuheben, dass Geographen das Côa-Tal als natürliche Grenze zwischen dem Zentralplateau der Meseta und den westlichen Gebirgen ansehen ${ }^{74}$.

Eine Vermutung zur Frage der Versorgung mit Silex aus weit entfernten Regionen besagt, dass Menschengruppen, die regelmäßig die biotischen und lithischen Ressourcen der beiden geographischen Regionen nutzten, sich im Rahmen ihrer saisonalen Wanderungen ebenso regelmäßig an den Fundorten des Côa-Tales getroffen haben könnten (Abb. 11). Demnach könnte das im Grenzbereich zweier geographischer Regionen gelegene Côa-Tal ein bevorzugter Ort des wirtschaftlichen und kulturellen Austausches gewesen sein - dies wäre eine Erklärung für die Notwendigkeit, graphische Symbole mit starkem Identitätswert herzustellen ${ }^{75}$.

\subsection{Was bedeuten die Freilandzeichnungen der Iberischen Halbinsel?}

Die Daten, die dank der Umsetzung eines systematischen Forschungsprojektes über die Freilandkunst des Côa-Tales und ihren Kontext gesammelt werden konnten, ermöglichen ein verbessertes Verständnis der Prozesse, die im Laufe der Zeit die Erhaltung der Petroglyphen bedingt haben und auch eine bessere Einschätzung der Repräsentativität der überlieferten Bilder. In Anbetracht der für die Iberische Halbinsel inzwischen klarer bestimmten Bedingungen und in Folge der Entdeckung pleistozäner Gravierungen im Nilbecken ${ }^{7}$ ist für die Zukunft mit einem exponentiellen Anwachsen der Entdeckung pleistozäner Freilandkunst zu rechnen.

Es ist nun möglich, den quantitativen Unterschied zwischen dem Bestand der Darstellungen im Freiland und dem der in Höhlen und Abris erhaltenen Gravierungen und Malereien zu relativieren, der zum Zeitpunkt der Entdeckung der ersten Anzeichen für die Existenz einer paläolithischen Freilandkunst hervorgehoben worden war77. Es bleibt jedoch weiterhin zu klären, ob das Fehlen zeichnerischer Äußerungen auf den unter freiem Himmel gelegenen Aufschlüssen der Karstregionen, in denen auch Bilderhöhlen vorkommen, das Ergebnis einer kulturell bedingten Auswahl oder die Folge zerstörender

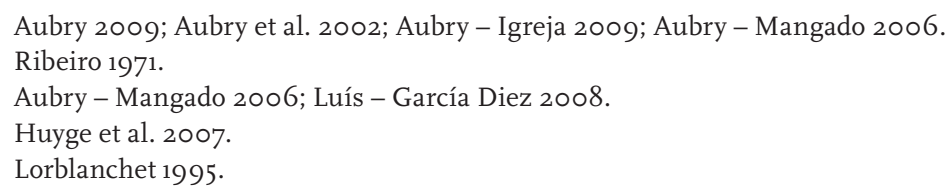


Verwitterungswirkungen ist, die auf den durch die Frostverwitterung stärker in Mitleidenschaft gezogenen Kalkfelsen nördlich der Pyrenäen ausgeprägter sind.

Im Hinblick auf die ältere Kunstphase im Côa-Tal deuten die verwendeten Techniken, der breite lineare Strich, die morphologische und stilistische Vereinfachung sowie die topographische Positionierung, darauf hin, dass - wie es sich auch durch unsere Raumanalyse der Fundorte Quinta da Barca und Penascosa bestätigt hat - ein klarer Wille vorhanden war, die Bilder auf möglichst breitem Raum den Blicken auszusetzen. Die kleineren und topographisch anders positionierten Ritzzeichnungen aus der Endphase des Jungpaläolithikums sind demgegenüber - trotz der Verwendung von Mehrfachlinien - deutlich schlechter sichtbar. Dieser Gegensatz ist Ausdruck unterschiedlicher Symbolgehalte, Funktionen, Umstände und Lesarten. Während die Kunst der älteren Phase eine Verknüpfung der Bilder im Maßstab der in ein Denkmal verwandelten Landschaft voraussetzt, scheint diejenige der jüngeren Phase auf den Maßstab des Felsens beschränkt zu sein.

Die Sichtbarkeitsanalyse zeigt, dass das Bestehen organisierter Parcours zwischen den Bildfeldern der älteren Phase ${ }^{78}$ auch - da sie untereinander nicht sichtbar sind - das Vorhandensein bestimmter durch die Darstellungen gelieferter Botschaften zur Orientierung sowie die Existenz eines Mittlers oder eines kollektiven Gedächtnisses bezüglich ihrer Standorte impliziert. Der Einwand, dass Darstellungen aus dazwischenliegenden Positionen eventuell nicht überliefert sind, lässt sich nicht ausräumen. Deshalb vertreten wir die alternative Vermutung, dass Bildfeldgruppen als szenographische Elemente gedient haben könnten, die Punkte umgeben haben, an denen Ereignisse von großer sozialer Bedeutung stattfanden. In diesem Sinne hat die Kulisse keine dekorative Funktion, sondern schafft einen Raum und nimmt an den darin ausgeführten Handlungen teil.

Die Vermutung einer Verbindung zwischen paläolithischer Kunst und sozialem Zusammenhalt ist nicht neu ${ }^{79}$. Mit Bezug auf die Fundstellen des Gravettien und des Solutréen ist auf regionaler Ebene das systematische Vorhandensein von aus $150 \mathrm{~km}$ Entfernung stammenden Silexstücken, nämlich aus in der Meseta und im lusitanischen Sedimentbecken gelegenen Rohstoffquellen, ein zusätzliches Argument sowohl für das Vorhandensein eines regelmäßigen Austausches zwischen den verschiedenen Menschengruppen dieser beiden Regionen als auch für diese erklärende Hypothese [Anm. d. Redaktion: die Verbindung der Bilder mit Funktionen im sozialen Gefüge] selbst ${ }^{8 \circ}$.

Die sozialen Praktiken heutiger Jäger- und Sammler-Gruppen - wie etwa der Einwohner der Andamanen-Inseln (Indischer Ozean) - können uns über den möglichen Zusammenhang der sozialen Verwendung dieser Kunst Auskunft geben. Zur Vermeidung territorialer Konflikte oder der Blutsverwandtschaft versammeln sich - in Regionen mit hohem demographischen Druck - Gruppen in angrenzenden oder umstrittenen Gebieten,

78 Baptista et al. 2008b; Baptista - Santos 2010.

79 Conkey 1980.

80 Aubry - Mangado 2006. 


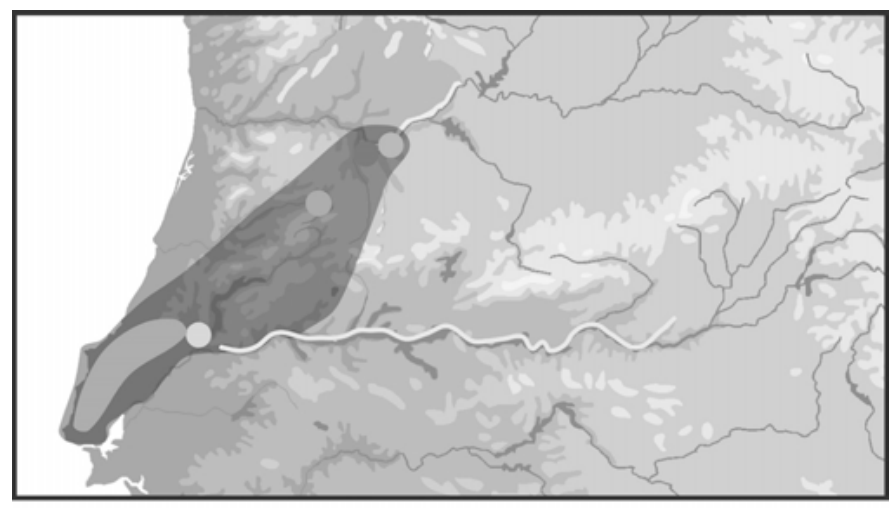

Fariseu (c4) - 10.800/11.800 BP

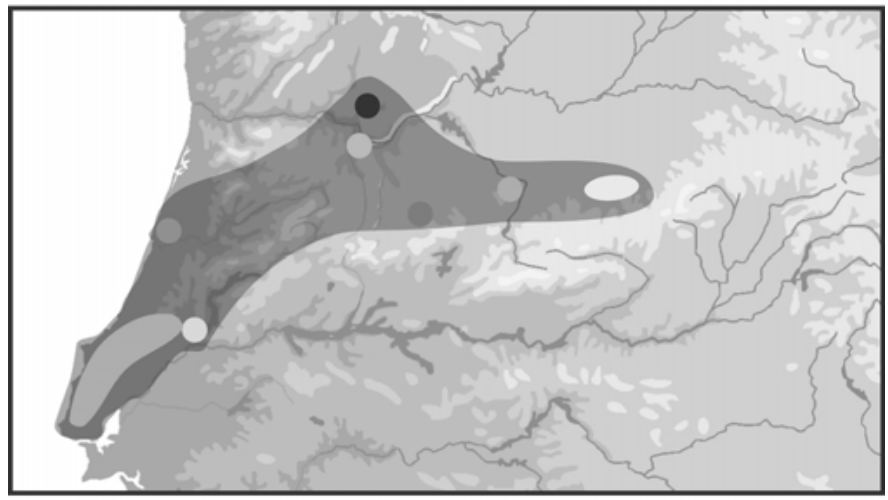

Olga Grande (c3) - 27.200/31.00 BP
Abb. 12 | Unterschiede hinsichtlich der Versorgung mit Silex und siliziumhaltigem Gestein zwischen Besiedlungsschichten des Endmagdalénien (Fariseu, Schicht 4) und des Gravettien (Olga Grande 4, Schicht 3).

um dort wirtschaftlichen Aktivitäten nachzugehen, gemeinsam zu essen, Geschenke auszutauschen, zu singen und zu tanzen oder gar Ehepartner auszutauschen und Kinder zu adoptieren ${ }^{81}$. Das Côa-Tal betreffend lässt sich die in einer geomorphologischen Grenzregion befindliche Kunst der älteren Phase unter einem derartigen Blickwinkel deuten.

In der Endphase des Jungpaläolithikums ist dagegen zu beobachten, dass die Bildfelder auf den geneigten Hängen verstreut und folglich in geringerem Umfang sichtbar sind. Ein Bruch wird auch durch die Untersuchung der Silexversorgung bestätigt, die eine Verringerung der Bedeutung der im Inneren der Iberischen Halbinsel gelegenen Rohstoffquellen anzeigt (Abb. 12). Dieser Wandel könnte eine Änderung der Bedeutung des Côa-Tales als Versammlungsort für Gruppen, die Territorien im Bereich der Küste und der Meseta nutzten, widerspiegeln ${ }^{82}$. Abgesehen von der Verbreitung innerhalb der Territorien ist ein weiterer grundlegender Unterschied [Anm. d. Übers.: gegenüber der

81 Kelly 2005.

82 Aubry - Mangado 2006. 
älteren Kunstphase] zu berücksichtigen: Das Auftreten von Kleinkunst in großer Menge wie es in Fariseu belegt ist - zu einer Zeit, in der ein Teil der Gravierungen der älteren Phase, die den Fundort umgeben, noch sichtbar war. Diese neue Form der Zeichnung auf teilweise verbrannten und absichtlich zerstörten, anscheinend zwischen den Überresten des täglichen Lebens zurückgelassenen Schiefertafeln scheint Bestandteil einer neuen Symbolik und sozialen Funktion zu sein.

\section{Bibliographie}

\section{Alcolea González - Balbín Behrmann 2006}

J. J. Alcolea González - R. Balbín Behrmann, Arte paleolítico al aire libre. El yacimiento rupestre de Siega Verde, Salamanca, Junta de Castilla y León Arqueología en Castilla y León 16 (Valladolid 2006).

\section{Aubry 1998}

T. Aubry, Olga Grande 4: uma sequência do Paleolítico superior no planalto entre o Rio Côa e a Ribeira de Aguiar, Revista Portuguesa de Arqueologia 1 (1), 1998, 5-26.

\section{Aubry 2001}

T. Aubry, L'occupation de la basse vallée du Côa pendant le Paléolithique supérieur, in: J. Zilhão - T. Aubry - A. F. Carvalho (Hgg.), Les premiers hommes modernes de la péninsule ibérique, Actes du Colloque de la Commission VIII de l’UISPP. Vila Nova de Foz Côa, 22-24 Octobre 1998, TrabArq 17 (Lisboa 2001) 253-273.

\section{Aubry 2009}

T. Aubry (Hg.), 200 séculos da história do Vale do Côa: incursões na vida quotidiana do caçadores-artistas do Paleolítico, TrabArq 52 (Lisboa 2009).

\section{Aubry - Baptista 2000}

T. Aubry - A. M. Baptista, Une datation objective de l'art du Côa, La Recherche Hors série 4, 2000, 54-55.

\section{Aubry - García Diez 2000}

T. Aubry - M. García Diez, Actualité sur la chronologie et l'interprétation de l'art de la vallée du Côa (Portugal). Les Nouvelles de l'Archéologie 82, 2000, 52-56. 


\section{Aubry - Igreja 2009}

T. Aubry - M. A. Igreja, Economy of Lithic Raw Material During the Upper Palaeolithic of the Côa Valley and the Sicó Massif (Portugal): Technological and Functional Perspectives, in: M. Araújo Igreja -I. Clemente Conte (Hgg.), Proceedings of the Workshop Functional Studies of Non Flint Stone Tools: Methodological Improvements and Archaeological Inferences (Lisbon, 23-25 may 2008), <http://arqant.imf.csic.es/aa/ index.php/en/elibrary.html> (29.03.2012).

\section{Aubry - Mangado Llach 2006}

T. Aubry - X. Mangado Llach, The Côa Valley (Portugal): From Lithic Raw Materials Characterization to the Reconstruction of Settlement Patterns during the Upper Palaeolithic, in: C. Bressy - A. Burke - P. Chalard - H. Marin (Hgg.), Notions de territoire et de mobilité. Exemples de l'Europe et des premières nations en Amérique du Nord avant le contact européen. Actes de sessions présentées au Xème Congrès annuel de l'E.A.A., 8-11.09.2004, Lyon (Liège 2006) 41-49.

\section{Aubry - Sampaio 2008}

T. Aubry - J. D. Sampaio, Fariseu: New Chronological Evidence for Open-Air Palaeolithic Art in the Côa Valley (Portugal), Antiquity 82 (316), 2008, <http://www.antiquity.ac.uk/ ProjGall/aubry/index.html> (15/03/2011).

\section{Aubry et al. 2002}

T. Aubry - X. Mangado Llach - F. Sellami - J. D. Sampaio, Open-Air Rock-Art Territories and Modes of Exploitation during the Upper Paleolithic in the Côa Valley (Portugal), Antiquity 76 (291), 2002, 62-76, <http://www.antiquity.ac.uk/ant/076/Anto760062. htm> (29.03.2012).

\section{Aubry et al. 2010}

T. Aubry - L. A. Dimuccio - M. Bergada - F. Sellami, F. 2010. Palaeolithic Engravings and Sedimentary Environments in the Côa River Valley (Portugal): Implications for the Detection, Interpretation and Dating of Open-Air Rock Art, JASc 37, 2010, 3306-3319.

Aubry et al. 2012

T. Aubry - L. Luís - L. A. Dimuccio, Nature vs. Culture: Present-Day Spatial Distribution and Preservation of Open-Air Rock Art in the Côa and Douro River Valleys (Portugal), Journal of Archaeological Science 39 (4), 2012, 848-866.

\section{Azéma 1992}

M. Azéma, La représentation du mouvement dans l'art animalier paléolithique des Pyrénées, Préhistoire Ariégoise 47, 1992, 19-76. 


\section{Bahn 1985}

P. Bahn, Ice Age Drawing on Open Rock Faces in the Pyrenees, Nature 313, 1985, 530-531.

\section{Balbín Behrmann - Alcolea González 2002}

R. de Balbín Behrmann - J. J. Alcolea González, L’art rupestre paléolithique à l’intérieur de la péninsule ibérique: une vision chrono-culturelle d'ensemble, in: D. Sacchi (Hg.), L'art paléolithique à l'air libre: le paysage modifié par l'image, Tautavel, Campôme, 7-9 octobre 1999 (Carcassone 2002) 139-157.

\section{Balbín Behrmann et al. I99I}

R. de Balbín Behrmann - J. J. Alcolea González - M. Santoja - R. Pérez Martín, Siega Verde (Salamanca): Yacimiento artístico paleolítico al aire libre, in: Del Paleolítico a la Historia. Ausstellungskatalog Salamanca (Salamanca 1991) 33-48.

\section{Baptista 1999}

A. M. Baptista, No Tempo sem Tempo: A arte dos caçadores paleolíticos do Vale do Côa (Vila Nova Foz Côa, Parque Arqueológico do Vale do Côa 1999).

\section{Baptista 200Ia}

A. M. Baptista, Ocreza (Evendos, Mação, Portugal Central): um novo sítio com arte paleolítica de ar livre, in: A. R. Cruz - L. Oosterbeek (Hgg.), Territórios, mobilidade e povoamento no alto Ribatejo II: Santa Cita e o Quaternário da região, Arkeos 11 (Tomar, CEIPHAR 2001) 163-192.

\section{Baptista 200Ib}

A. M. Baptista, The Quaternary Rock Art of the Côa Valley, in: J. Zilhão - T. Aubry A. F. Carvalho (Hgg.), Les premiers hommes modernes de la péninsule ibérique, Actes du Colloque de la Commission VIII de l'UISPP. Vila Nova de Foz Côa, 22-24 Octobre 1998, TrabArq 17 (Lisboa 2001) 237-252.

\section{Baptista 2004}

A. M. Baptista, Arte paleolítica de ar livre no rio Zêzere (Barroca, Fundão), Ebvrobriga 1, 2004, 9-16.

\section{Baptista 2008}

A. M. Baptista, Aspecto da Arte Magdalenense e Tardiglaciar no Vale do Côa, in:

A. T. Santos - L. Luís (Hgg.), Do Paleolítico à Contemporaneidade, Actas do Fórum Valorização e Promoção do Património Regional 3 (Porto 2008) 16-31, <http://www.artecoa.pt/Ficheiros/Bibliografia/1523/1523.pt.pdf> (29.03.2012). 


\section{Baptista 2009}

A. M. Baptista, O paradigma perdido: O Vale do Côa e a arte paleolítica de ar livre em Portugal (Porto 2009).

\section{Baptista - García Diez 2004}

A. M. Baptista, A.M.; García Diez, M. 2002. L'art paléolithique dans la vallée du Côa (Portugal): la symbolique dans l'organisation d'un sanctuaire de plein air, in: D. Sacchi (Hg.), L'art paléolithique à l'air libre: le paysage modifié par l'image, Tautavel, Campôme, 7-9 octobre 1999 (Carcassone 2002) 187-205.

\section{Baptista - Gomes I995}

A. M. Baptista - M. V. Gomes, Arte rupestre do Vale do Côa 1: Canada do Inferno: Primeiras impressões. TrabAntrEtn 35 (4), 1995, 349-422.

\section{Baptista - Reis 2008}

A. M. Baptista - M. Reis, Prospecção da arte rupestre no Vale do Côa e Alto Douro portugés: Ponto da situação em julho de 2006, in: R. de Balbín Behrmann (Hg.), Arte al aire libre en el Sur de Europa (Valladolid 2008) 145-192.

\section{Baptista - Santos 2010}

A. M. Baptista - A. T. Santos, Confronting Two Sceneries on the Same Stage: from Gravettian-Solutrean to Magdalenian in Penascosa/Quinta da Barca (Vila Nova de Foz Côa, Portugal, in: A. M. S. Bettencourt - M. J. Sanches - L. B. Alves - R. Fábregas Valcarce (Hgg.), Conceptualising Space and Place on the Role of Agency. Memory and Identity in the Construction of Space from the Upper Palaeolithic to the Iron Age in Europe, BARIntSer 2058 (Oxford 2010) 61-69.

\section{Baptista et al. 2006}

A. M. Baptista - A. T. Santos - D. Correia, Da ambiguidade das margens na Grande Arte de ar livre no Vale do Côa: Reflexões em torno da organização espacial do santuário Gravetto-Solutrense na estação da Penascosa/Quinta da Barca, Côavisão 8, 2006 , $156-184$.

\section{Baptista et al. 2008a}

A. M. Baptista - A. T. Santos - D. Correia, Estruturação simbólica da arte GravettoSolutrense em torno do monte do Fariseu (Vale do Côa). in: A. T. Santos - J. Sampaio (Hgg.), Pré-história: Gestos intemporais. III Congresso de Arqueologia de Trás-osMontes, Alto Douro e Beira Interior, Actas das sessões 1 (Porto 2008) 38-61. 


\section{Baptista et al. 2008b}

A. M. Baptista - A. T. Santos - D. Correia, O santuário arcaico do Vale do Côa: novas pistas para a compreensão da estruturação do Bestiário Gravettense e/ou gravettosolutrense, in: R. de Balbín Behrmann (Hg.), Arte al aire libre en el Sur de Europa (Valladolid 2008) 89-144.

\section{Bednarik I995}

R. G. Bednarik, The Côa Petroglyphs: an Obituary to the Stylistic Dating of Palaeolithic Rock-Art, Antiquity 69, 1995, 877-882.

\section{Cailleux 1979}

A. Cailleux, Notice sur le code des couleurs des sols (Paris 1979).

Chauvière et al. 2009

F. X. Chauvière - S. Tymula - A. Calame - I. Dechanez, Conservation et évolution des surface rocheuses gravées et piquetées de la Vallée du Côa : les données du projet »Quinta da Barca Sul«, in: T. Aubry (Hg.), 200 séculos da história do Vale do Côa: incursões na vida quotidiana do caçadores-artistas do Paleolítico, TrabArq 52 (Lisboa 2009) 443-477.

\section{Collado 2009}

H. Collado, Arte rupestre prehistórico en Extremadura 1997-2006, in: R. de Balbín Behrmann (Hg.), Arte al aire libre en el Sur de Europa (Valladolid 2008) 287-322.

\section{Conkey 1980}

M. Conkey, The Identification of Prehistoric Hunter-Gatherer Aggregation Sites:

The Case of Altamira, Current Anthropology 21, 1980, 609-630.

\section{Couraud 1985}

C. Couraud, L'art azilien: origine, survivance (Paris 1985).

\section{D'Errico 1994}

F. D’Errico, L’art gravé azilien: de la technique à la signification, Gallia Préhistoire Suppl. 31 (Paris 1994).

\section{Dorn 1997}

R. Dorn, Constraining the Age of the Côa Valley (Portugal) Engraving with Radiocarbon Dating, Antiquity 71, 1997, 105-115. 


\section{Fernandes 2010}

A. P. B. Fernandes, Slope Orientation of Rock Art Sites in the Côa Valley, Portugal:

A Case Study in the Spatial Distribution of Open-Air Upper Palaeolithic Rock Art, in: Congrès de l'IFRAO, septembre 2010 - Symposium, L'art pléistocène en Europe (PréActes), <http://www.arte-coa.pt/Ficheiros/Bibliografia/1329/1329.pt.pdf> (29.03.2012).

\section{García Diez - Aubry 2002}

M. García Diez - T. Aubry, Grafismo mueble en el Valle del Côa (Vila Nova de Foz Côa, Portugal): la estación arqueológica de Fariseu, Zephyrus 55, 2002, 157-182.

\section{García Diez - Luís 2003}

M. García Diez - L. Luís, José Alcino Tomé e o último ciclo artístico rupestre do Vale do Côa: um caso de etnoarqueologia, Estudos Pré-Históricos 10-11, 2003, 199-223.

\section{García Diez et al. 2009}

M. García Diez - T. Aubry - J. D. Sampaio, Los materiales colorantes en los yacimientos pleistocenos del Valle de Côa: Quinta da Barca Sul, Olga Grande 4 y Cardina I, in: T. Aubry (Hg.), 200 séculos da história do Vale do Côa: incursões na vida quotidiana do caçadores-artistas do Paleolítico, TrabArq 52 (Lisboa 2009) 395-435.

\section{Guy 2010}

E. Guy, Préhistoire du sentiment artistique: L'invention du style, il y a 20 o० ans (Bruxelles 2010).

\section{Huyge et al. 2007}

D. Huyge - M. Aubert - H. Barnard - W. Claes - J. C. Darnell - M. de Dapper - E. Figari S. Ikram - A. Lebrun-Nélis - I. Therasse, »Lascaux along the Nile«: Late Pleistocene rock art in Egypt, Antiquity 81 (313), 2007, <http://antiquity.ac.uk/projgall/huyge/index.html> (29.03.2012).

\section{Jorge et al. I98I}

S. O. Jorge - V. O. Jorge - C. A. F. Almeida - M. J. Sanches - M. T. Soeiro, Gravuras rupestres de Mazouco (Freixo de Espada-à-Cinta), Arqueologia 3, 1981, 3-12.

\section{Kelly 2005}

R. C. Kelly, The Evolution of Lethal Intergroup Violence, Proceedings of the National Academy of Sciences 102 (43), 2005, 15294-15298. 


\section{Leroi-Gourhan 1992}

A. Leroi-Gourhan, L'espace et le temps dans l'art pariétal paléolithique, in: A. Leroi-

Gourhan, L'art pariétal: Langage de la préhistoire. Essays previously published 1958-1982 (Grenoble 1992) 259-271.

\section{Lorblanchet 1995}

M. Lorblanchet, Les grottes ornées de la préhistoire: Nouveaux regards (Paris 1995).

\section{Luís 2008}

L. Luís, A Arte e os Artistas do Vale do Côa (Vila Nova de Foz Côa, Parque Arqueológico do Vale do Côa; Associação de Municípios do Vale do Côa 2008).

\section{Luís 2009}

L. Luís, »Per petras et per signos «: A arte rupestre do Vale do Côa enquanto construtora do espaço na Proto-história, in: P. J. Sanabria Marcos (Hg.), Lusitanos y vettones: Los pueblos prerromanos en la actual demarcación Beira Baixa - Alto Alentejo - Cáceres, Memorias 9 (Cáceres 2009) 213-240.

\section{Luís - García Díez 2008}

L. Luís - M. García Díez, Same Tradition, Different Views: The Côa Valley Rock Art and Social Identity, in: I. Domingo Sanz - D. Fiore - S. K. May (Hgg.), Archaeologies of Art: Time, Place and Identity, One World Archaeology Series 55 (Wallnut Creek 2008) 151-170.

\section{Mercier et al. 200I}

N. Mercier - H. Valladas - L. Froget - J. L. Jorons - J. L. Reyss - T. Aubry, Application de la méthode de la thermoluminescence à la datation des occupations paléolithiques de la vallée du Côa, in: J. Zilhão - T. Aubry - A. F. Carvalho (Hgg.), Les premiers hommes modernes de la péninsule ibérique, Actes du Colloque de la Commission VIII de l'UISPP. Vila Nova de Foz Côa, 22-24 Octobre 1998, TrabArq 17 (Lisboa 2001) 275-280.

\section{Mercier et al. 2006}

N. Mercier - H. Valladas - T. Aubry - J. Zilhão - J. L. Jorons - J. L. Reyss - F. Sellami, Fariseu: First Confirmed Open-Air Palaeolithic Parietal Art Site in the Côa Valley (Portugal), Antiquity 80 (310), 2006, <http://antiquity.ac.uk/projgall/mercier/index.html> $(29.03 .2012)$.

\section{Otte 2002}

M. Otte, Le rapport à l'espace aérien dans l'art préhistorique, in: D. Sacchi (Hg.), L'art paléolithique à l'air libre: le paysage modifié par l'image, Tautavel, Campôme, 7-9 octobre 1999 (Carcassone 2002) 177-180. 


\section{Plisson 2009}

H. Plisson, Analyse tracéologique de 4 pics d'Olga Grande: des outils pour les gravures de plein air?, in: T. Aubry (Hg.), 200 séculos da história do Vale do Côa: incursões na vida quotidiana do caçadores-artistas do Paleolítico, TrabArq 52 (Lisboa 2009) 436-443.

\section{Rebanda 1995}

N. Rebanda, Os trabalhos arqueológicos e o complexo de arte rupestre do Côa (Lisboa 1995).

\section{Ribeiro I97I}

O. Ribeiro, Publicações recentes acerca da Península Ibérica: Quarta notícia. Finisterra 12, 1971, 255-273.

\section{Ribeiro 200I}

M. L. Ribeiro, Carta geológica simplificada do parque Arqueológico do Vale do Côa, esc: 1/80 o०o: Notícia explicativa (Vila Nova de Foz Côa, Parque Arqueológico do Vale do Côa 2001).

\section{Ripoll López - Municio González 1992}

S. Ripoll López - L. J. Municio González, Las representaciones de estilo paleolítico en el conjunto de Domingo García (Segovia), Espacio, Tiempo y Forma. Serie 1: Prehistoria y Arqueología 5, 1992, 107-138.

\section{Sacchi et al. 1988}

D. Sacchi - J. Abelanet - J. L. Brulé - Y. Massiac - C. Rubiella - P. Vilette, Les gravures rupestres de Fornols-Haut, Pyrénées-Orientales, L’Anthropologie 92 (1), 1988, 87-100.

\section{Schefer 1999}

J. L. Schefer, Questions d'art paléolithique (Paris 1999).

\section{Valladas et al. 20oI}

H. Valladas - N. Mercier - L. Froget - J. L. Jorons - J. L. Reyss - T. Aubry, TL Dating of Upper Palaeolithic Sites in the Côa Valley (Portugal), Quaternary Science Reviews 20 (5-9), 2001, 939-943.

\section{Watchman 1995}

A. Watchman, A. 1995. Recent Petroglyphs, Foz Côa, Rock Art Research 12 (2), 1995, 104-108.

\section{Watchman 1996}

A. Watchman, A Review of the Theory and Assumption in the AMS Dating of the Foz Côa Petroglyphs, Portugal, Rock Art Research 13 (1), 1996, 21-30. 


\section{Wheatley - Gillings 2002}

D. Wheatley - M. Gillings, Spatial Technology and Archaeology: The Archaeological Applications of GIS (London 2002).

\section{Zilhão 1995}

J. Zilhão, The Age of the Côa Valley (Portugal) Rock-Art: Validation of Archaeological

Dating to the Palaeolithic and Refutation of »Scientific« Dating to Historic or ProtoHistoric Times, Antiquity 69, 1995, 883-901.

\section{Zilhão 1997}

J. Zilhão (Hg.), Arte rupestre e Pré-história do Vale do Côa: trabalhos de 1995-1996 (Lisboa 1997).

\section{Zilhão I998}

J. Zilhão, The Rock Art of the Côa Valley, Portugal: Significance, Conservation and Management, Conservation and Management of Archaeological Sites 2, 1998,193-206.

\section{Zilhão 2003}

J. Zilhão, Vers une chronologie plus fine du cycle ancien de l'art paléolithique de la Côa: quelques hypothèses de travail, in: R. de Balbín - P. Bueno Ramírez (Hgg.), El arte prehistórico desde los inicios del siglo XX: Primer symposium internacional de arte prehistórico de Ribadesella (Ribadesella 2003) 75-90.

\section{Abbildungsnachweis}

Abb. 1-12: T. Aubry - L. Luís; Tab. 1-4: T. Aubry - L. Luís. 
